\title{
Structure- Kinetics Correlations in Isostructural Crystals of $\alpha$-(ortho-tolyl)-Acetophenones: Pinning Down Electronic Effects Using Laser-Flash Photolysis in the Solid State
}

Anoklase J.-L. Ayitou, ${ }^{a}$ Kristen Flynn, ${ }^{a}$ Steffen Jockusch, ${ }^{b}$ Saeed I. Khan ${ }^{a}$ and Miguel A. Garcia-Garibay ${ }^{a *}$ ${ }^{a}$ Department of Chemistry and Biochemistry, University of California Los Angeles, Los Angeles, CA 90095, USA

${ }^{b}$ Department of Chemistry, Columbia University, New York, NY 10027, USA

e-Mail: mgg@chem.ucla.edu

TABLE OF CONTENTS

S2. GENERAL METHODS

S3. LASER FLASH PHOTOLYSIS (LFP) OF 1a-c IN ACETONITRILE AND IN CRYSTALLINE SUSPENSION

S8. SYNTHETIC PROTOCOLS: Synthetic protocol for $\alpha$-tolylacetophenones 1a-c

S10. POWDER XRD CRYSTALLOGRAPHY OF 1a-c

S11. GENERAL IRRADIATION PROCEDURES AND CHARACTERIZATION OF PHOTOPRODUCTS 2a,b

Solution phase irradiation

Crystalline phase irradiation

Characterization of photoproducts $\mathbf{2 a , b}$

S12. DYNAMIC LIGHT SCATTERING ANALYSIS OF CRYSTALLINE SUSPENSIONS OF 1a-c and SEM of $1 \mathrm{c}$.

S16. SPECTRAL DATA FOR 1a-c

S31. REFERENCES 


\section{GENERAL METHODS}

All commercially obtained reagents/solvents were used as received without further purification. Unless stated otherwise, reactions were conducted in oven-dried glassware under argon atmosphere. ${ }^{1} \mathrm{H}-\mathrm{NMR}$ and ${ }^{13} \mathrm{C}-\mathrm{NMR}$ spectra were recorded on Bruker $400 \mathrm{MHz}(100 \mathrm{MHz}$ for

$\left.{ }^{13} \mathrm{C}\right)$ spectrometers. Data from the ${ }^{1} \mathrm{H}-\mathrm{NMR}$ spectroscopy are reported as chemical shift ( $\left.\delta \mathrm{ppm}\right)$ with the corresponding integration values. Coupling constants $(J)$ are reported in hertz $(\mathrm{Hz})$. Standard abbreviations indicating multiplicity were used as follows: s (singlet), b (broad), d (doublet), $\mathrm{t}$ (triplet), q (quartet), $\mathrm{m}$ (multiplet) and virt (virtual). Data for ${ }^{13} \mathrm{C} N \mathrm{NM}$ spectra are reported in terms of chemical shift $(\delta \mathrm{ppm})$. High-resolution mass spectrum data were recorded on a DART spectrometer in positive (ESI+) ion mode. UV-Vis absorption and transmission spectra were recorded on Ocean Optics spectrometer (DT-MINI-2-GS UV-VIS-NIR LightSource and USB2000+ using SpectraSuite software package); a Transmission Dip-Probe (TP300-UVVIS) was used to measure the \%transmittance of the crystalline suspensions. Dynamic Light Scattering (DLS) data were recorded using a Beckman-Coulter N4 Plus particle analyzer with a $10 \mathrm{~mW}$ helium-neon laser at $632.8 \mathrm{~nm}$. The particle size was determined using the $62.6^{\circ}$ detection angle and was calculated using the size distribution processor (SDP) analysis package provided by the manufacturer. Melting point values were recorded on a Melt-Point II ${ }^{\circledR}$ apparatus. Infra-Red spectra were recorded on a PerkinElmer ${ }^{\circledR}$ Spectrum 100 spectrometer equipped with a universal ATR sampling accessory. Nanosecond transient absorption experiments were performed using Laser Flash Photolysis instrument from Edinburgh Instruments in conjunction with a Nd:YAG laser (Brilliant b, Quantel®) with 266-nm output, 4-6 ns pulse width and 36-40 $\mathrm{mJ}$ pulse energy. The optical detection is based on a pulsed Xenon arc lamp (450 W), a monochromator (TMS300, Czerny-Turner), a photomultiplier detector (Hamamatsu R928) and a digital oscilloscope (TDS3012C, $100 \mathrm{MHz}$ and $1.25 \mathrm{GS} / \mathrm{s}$ from Tektronix). The laser flash photolysis experiments were performed with $1 \mathrm{~cm}$ quartz flow cell mounted on a home-built sample holder that is placed at the cross-section of the laser incident beam and the probe light. Continuously $\mathrm{N}_{2}$ gas purged $\mathrm{ACN}$ solutions or crystalline suspensions of ketones were flown through the quartz cell using a peristaltic pump (Masterflex L/S) at a rate of 2.5-4 $\mathrm{mL} / \mathrm{min}$. Time-resolved absorption maps were recorded with continuous flow of samples through the quartz cell. Lifetimes at $\lambda_{\max }$ for end-of-pulse spectra were reproducible and doubly verified/processed with Edinburgh Instruments L900 internal software and Igor Pro (version 6.34A, Wavemetrics) software. GC analyses were performed using HP 5835 and Agilent 6890 instruments. 
<smiles>Cc1ccccc1CC(=O)c1ccccc1</smiles>

$1 \mathrm{a}$<smiles>OC1(c2ccccc2)Cc2ccccc2C1</smiles>

2a<smiles>Cc1ccc(C(=O)Cc2ccccc2C)cc1</smiles>

$1 b$<smiles>Cc1ccc(CC(=O)c2ccccc2)cc1</smiles>

1c<smiles>Cc1ccc(C2(O)Cc3ccccc3C2)cc1</smiles>

Chart S1. $\alpha$-tolylacetophenones 1 and corresponding 2-indanols photoproducts $\mathbf{2 a}$ and $\mathbf{2 b}$.

\section{LASER FLASH PHOTOLYSIS (LFP) OF 1a-c}

MeCN and nanocrystalline suspension sample preparation

Solution phase samples were prepared by dissolving freshly recrystallized compounds 1a-c in spectrophotometric/HLPC grade acetonitrile such that the O.D. for each sample reads $\approx 0.3$ at $266 \mathrm{~nm}$.

Nanocrystalline suspensions were prepared via the reprecipitation of organic solution of ketones 1a-c in MeCN into purified Millipore water followed by passivation of the nanocrystals with cetrimonium bromide (CTAB) solution in order to have stable suspensions.

For LFP experiments, the crystalline suspensions were prepared by injecting a $8.5-12.5 \mathrm{mM}$ acetonitrile solution of the desired ketone into 50-150 times the volume of purified vortexing Millipore water followed by addition of equal volume of CTAB solution $(0.4 \mathrm{mM})$ in order to have an O.D. $\approx 0.3-0.4$.

DLS experiments: The crystalline suspensions were prepared by injecting a 18-24 mM solution of the desired ketone into 35-100 times the volume of purified vortexing Milliport water followed by addition of equal volume of CTAB solution $(0.4 \mathrm{mM})$. 
UV-Vis spectra of compounds $1 \mathrm{a}-\mathrm{c}$ in $\mathrm{MeCN}$ and as nanocrystalline suspension
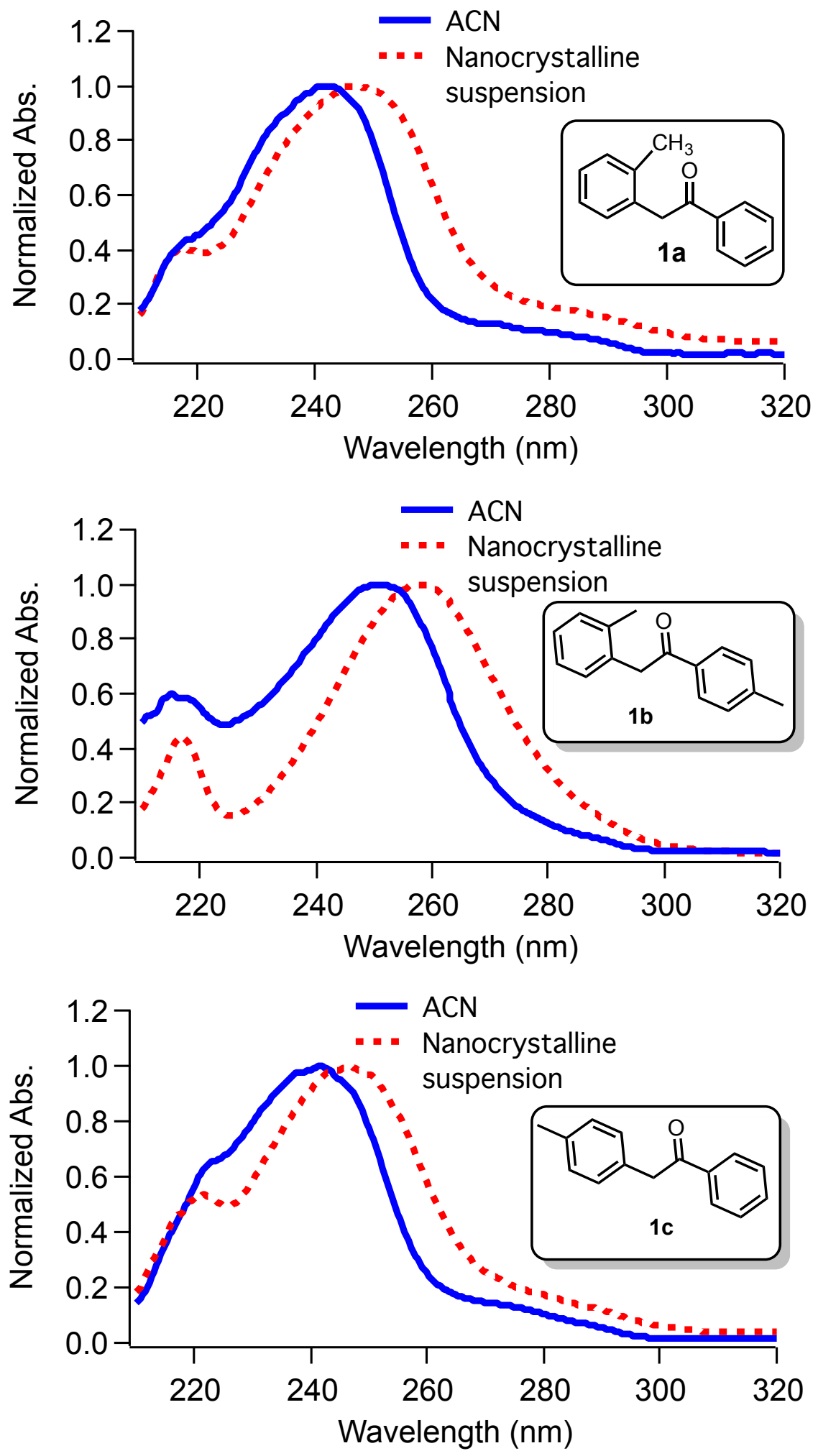

Figure S1: UV-vis spectra of compounds 1a-c in MeCN and as nanocrystalline suspension. 
Transient absorption spectra and transient kinetics for compounds 1a-c

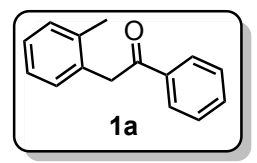

Time-resolved transient absorption
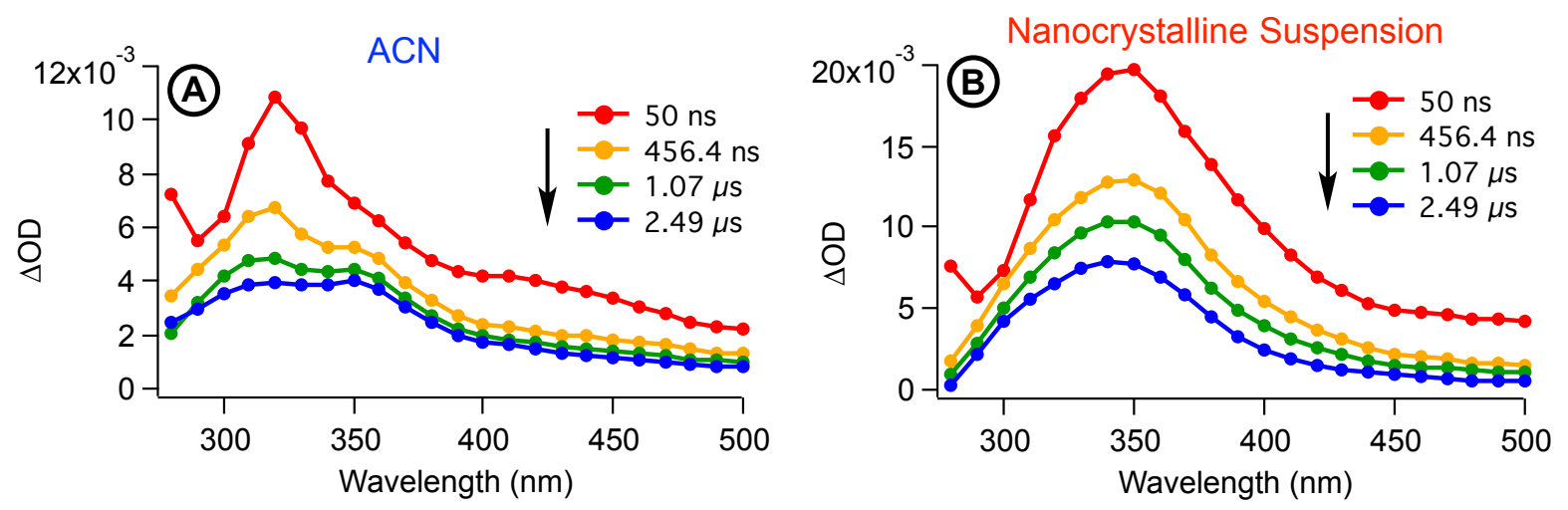

(C) Transient kinetics monitored

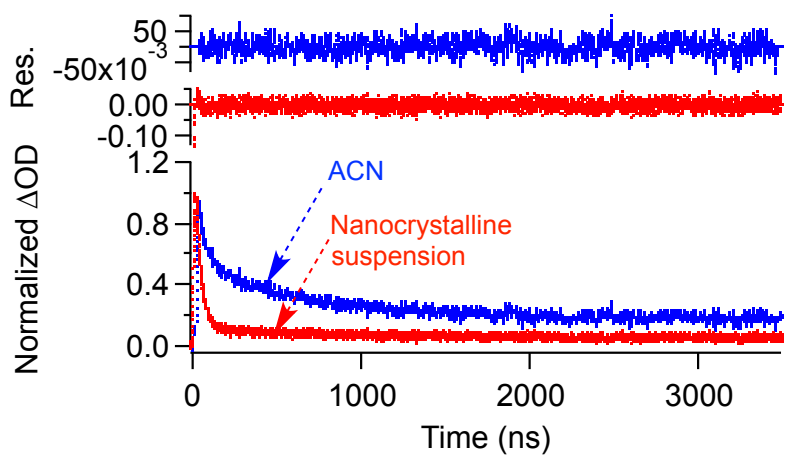

Figure S2: A) Time-resolved transient absorption spectra of 1a in acetonitrile. B) Time-resolved transient absorption spectra of $\mathbf{1 a}$ in the nanocrystalline phase. C) Decay kinetics detected in acetonitrile $\left(\lambda_{\max }=320 \mathrm{~nm}\right)$ with bi-exponential fit residuals and in nanocrystalline suspension $\left(\lambda_{\max }=340 \mathrm{~nm}\right)$ with single-exponential fit residuals.

Table S1: Fit decay kinetrics data for $1 \mathrm{a}$

\begin{tabular}{|cc|cc|}
\hline \multicolumn{2}{|c|}{$\boldsymbol{\tau}_{\text {Triplet State }}(\mathbf{n s})[\mathbf{A}]^{\mathrm{a}}$} & \multicolumn{2}{c|}{$\boldsymbol{\tau}_{\mathbf{1 , 5 - B i r a d i c a l}}$ (ns) $[\mathbf{A}]^{\mathrm{a}}$} \\
\hline ACN & $\begin{array}{c}\text { Nanocrystalline } \\
\text { suspension }\end{array}$ & ACN & $\begin{array}{c}\text { Nanocrystalline } \\
\text { suspension }\end{array}$ \\
\hline 6 & 37 & $\begin{array}{c}50[0.41] \text { and } \\
650[0.35]^{\mathrm{b}}\end{array}$ & n.a. \\
\hline
\end{tabular}

${ }^{a}$ Pre-exponential factor. ${ }^{\mathrm{b}}$ Decay fit to a double exponential. 


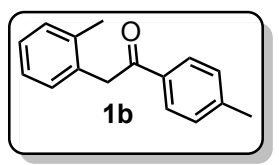

Time-resolved transient absorption
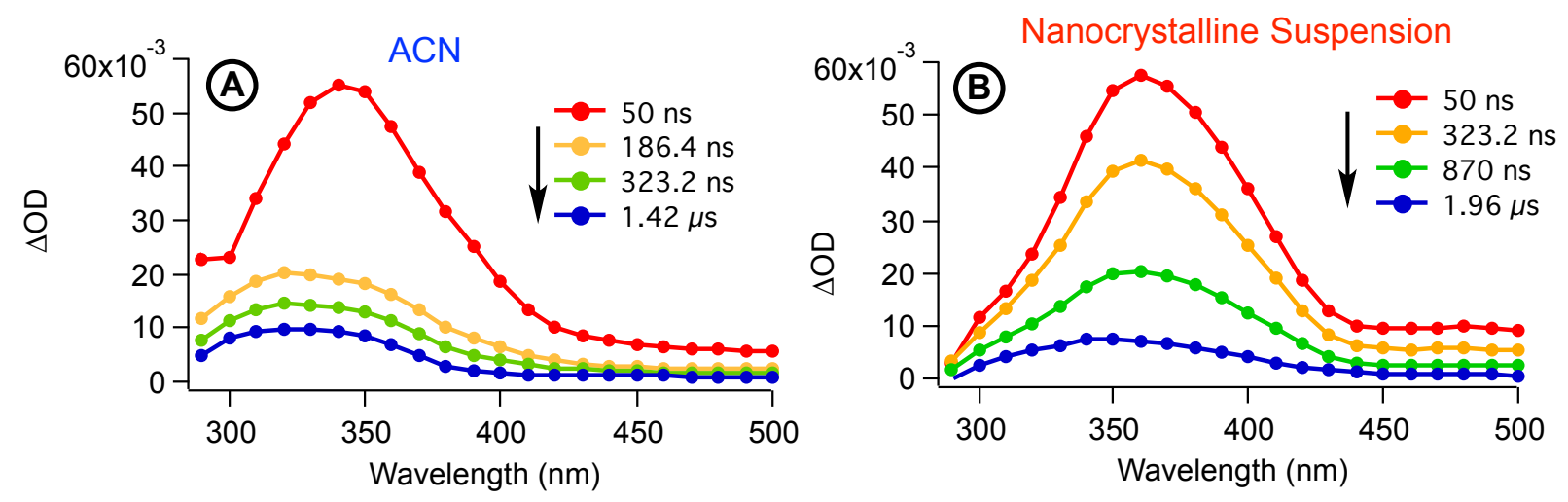

(B) Transient kinetics monitored

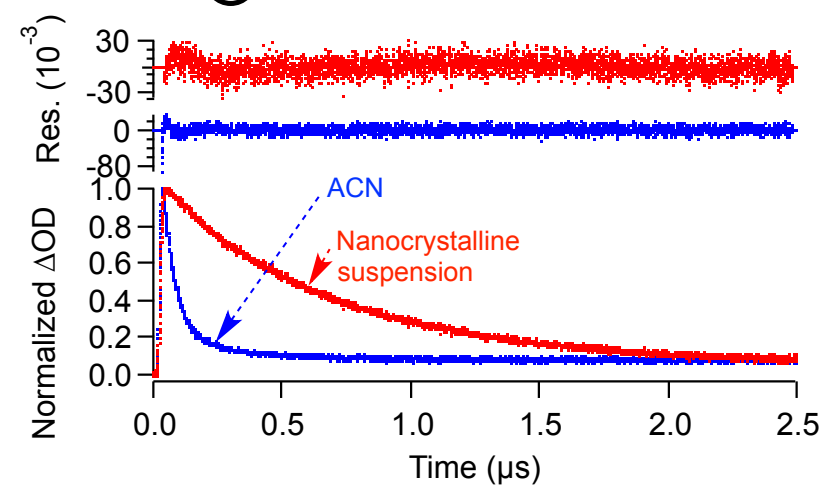

Figure S3: A) Time-resolved transient absorption spectra of $\mathbf{1 b}$ in acetonitrile. B) Time-resolved transient absorption spectra of $\mathbf{1 b}$ in the nanocrystalline phase. C) Decay kinetics detected in acetonitrile $\left(\lambda_{\max }=340 \mathrm{~nm}\right)$ with bi-exponential fit residuals and in nanocrystalline suspension $\left(\lambda_{\max }=360 \mathrm{~nm}\right)$ with single-exponential fit residuals.

Table S2: Fit decay kinetrics data for $1 \mathrm{~b}$

\begin{tabular}{|cc|}
\hline \multicolumn{2}{|c|}{$\boldsymbol{\tau}_{\text {Triplet State }}(\mathbf{n s})[\mathbf{A}]^{\mathrm{a}}$} \\
\hline ACN & $\begin{array}{c}\text { Nanocrystalline } \\
\text { suspension }\end{array}$ \\
\hline 70 & 660 \\
\hline$(50[0.8] \text { and } 309[0.12])^{\mathrm{b}}$ & \\
\hline
\end{tabular}

${ }^{a}$ Pre-exponential factor. ${ }^{b}$ Decay fit to a double exponential. 


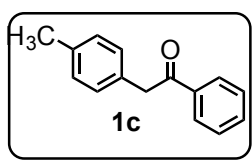

Time-resolved transient absorption
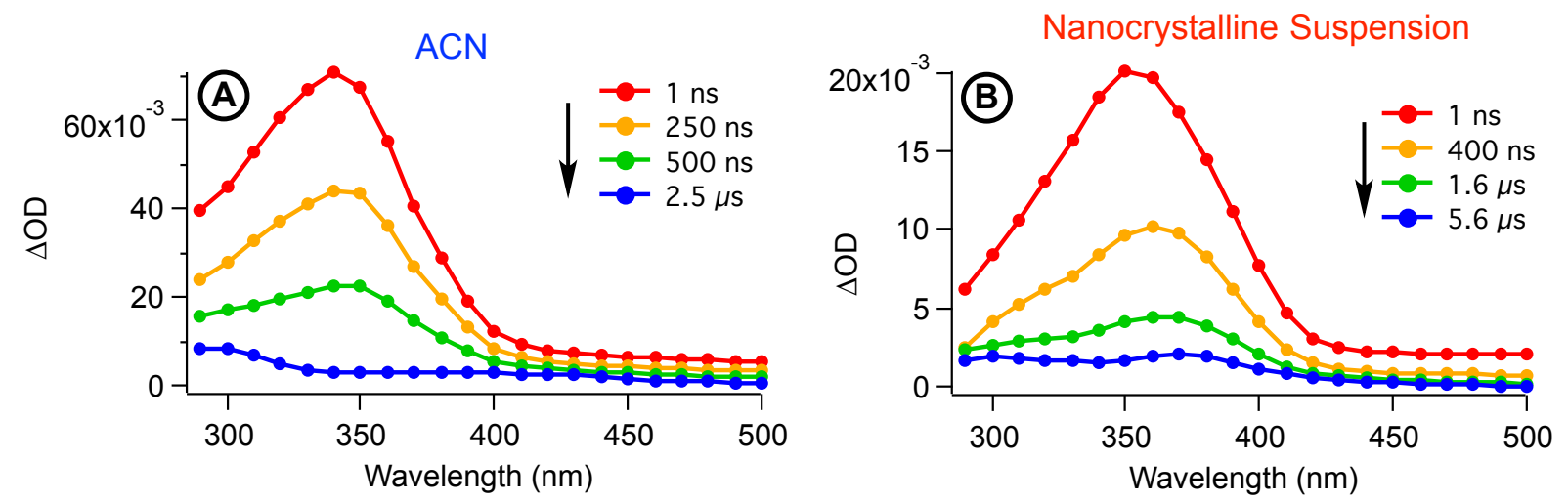

(C) Transient kinetics monitored at $340 \mathrm{~nm}$

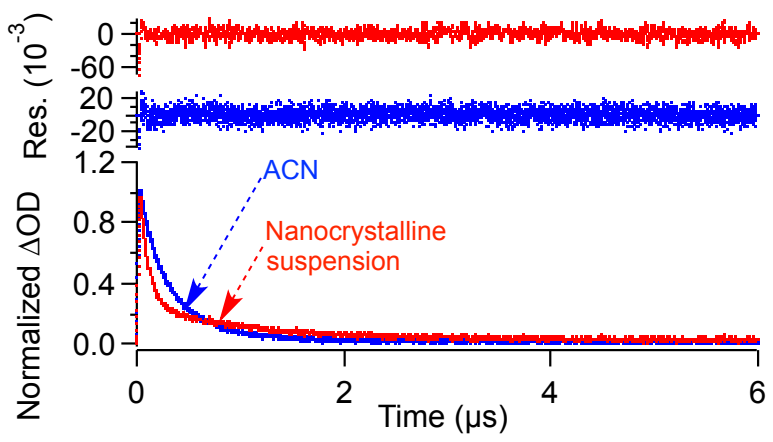

Figure S4: A) Time-resolved transient absorption spectra of 1c in acetonitrile. B) Time-resolved transient absorption spectra of 1c in the nanocrystalline phase. C) Decay kinetics detected in acetonitrile (with single-exponential fit residuals) and in nanocrystalline suspension at $\lambda_{\max }=340$ $\mathrm{nm}$ (with bi-exponential fit residuals).

Table S3: Fit decay kinetrics data for 1c

\begin{tabular}{|cc|}
\hline & $\tau_{\text {Triplet State }}(\mathbf{n s})[\mathrm{A}]^{\mathrm{a}}$ \\
\hline ACN & $\begin{array}{c}\text { Nanocrystalline } \\
\text { suspension }\end{array}$ \\
\hline 308 & $88[0.78]$ and $1030[0.22]^{\mathrm{b}}$ \\
\hline
\end{tabular}

${ }^{a}$ Pre-exponential factor. ${ }^{b}$ Decay fit to a double exponential. 
SYNTHETIC PROTOCOLS: Synthetic protocol for $\alpha$-tolylacetophenones 1a-c

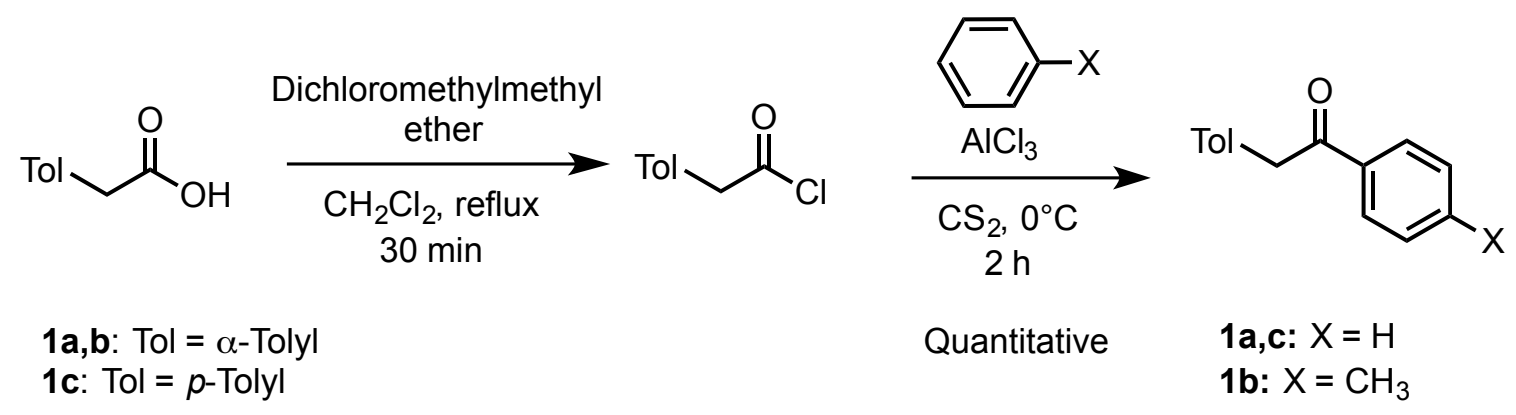

Scheme S1. Synthesis of $\alpha$-tolylacetophenones 1a-c.

Compounds 1a, 1b, 1c were synthesized using the standard protocol of Friedel-Crafts acylation (Scheme S1). In a typical reaction, 2-arylacetic acid (1 equiv.) was converted to the corresponding acid chloride using dichloromethyl methyl ether (2.5 equiv.). The acid chloride prepared in situ was concentrated under reduced pressure and dried under high vacuum to remove traces of methylene chloride and dichloromethyl methyl ether. Afterwards, a phenylene reagent (10 equiv.) was added to the freshly prepared acid chloride in carbon disulfide at $0^{\circ} \mathrm{C}$; this mixture was stirred at $0^{\circ} \mathrm{C}$ followed by portionwise additions of $\mathrm{AlCl}_{3}$ (3 equiv.) over $30 \mathrm{~min}$. The new mixture was allowed to react for an additional $2 \mathrm{~h}$ (Scheme 1). Afterwards, the reaction was slowly quenched using cold water and the expected compound was extracted from the aqueous mixture using ethyl acetate. The combined organic fractions were dried over anh. $\mathrm{Na}_{2} \mathrm{SO}_{4}$ and concentrated under reduced pressure. Compounds $1 \mathrm{a}-\mathrm{c}$ were purified by flash chromatography on silica gel (solvent system: 5 - $10 \%$ EtOAc:hexanes) to afford crystalline/powdered white solids with yields $>98 \%$ except in the case of 1 b (59\% yield), where about $30 \%$ of the ortho regiomer was also isolated. 
Characterization (NMR, ATR-FTIR, Melting Point) of 1a-c

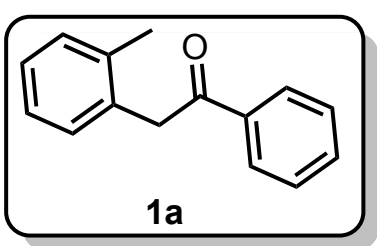

${ }^{1} \mathrm{H}$ NMR (400 MHz, $\left.\mathrm{CDCl}_{3}, \delta\right): 2.29$ (s, 3H, CH$\left.)_{3}\right), 4.33\left(\mathrm{~s}, 2 \mathrm{H}, \mathrm{CH}_{2}\right.$ ), $7.16-7.23(\mathrm{~m}, 4 \mathrm{H}), 7.48-7.52(\mathrm{t}, \mathrm{J}=8 \mathrm{~Hz}, 2 \mathrm{H}, \mathrm{Ar}-\mathrm{H}), 7.58-7.62(\mathrm{t}$, $J=8 \mathrm{~Hz}, 1 \mathrm{H}, \mathrm{Ar}-\mathrm{H}), 8.04-8.06(\mathrm{~d}, J=8 \mathrm{~Hz}, 2 \mathrm{H}, \mathrm{Ar}-\mathrm{H})$.

${ }^{13} \mathrm{C} \mathrm{NMR}\left(100 \mathrm{MHz}, \mathrm{CDCl}_{3}, \delta\right): 19.8,43.5,126.1,127.27,129.4,129.7$, 130.3, 130.4, 133.2, 133.5, 136.9, 137.0, 197.5 .

ATR-FTIR: 2899.61, 1683.83, 1593.40, 1579.80, 1446.95, 1336.78, 1210.44, 1170.13, 996.84, $751.40,742.92,688.80 \mathrm{~cm}^{-1}$

HRMS: $\mathrm{C}_{15} \mathrm{H}_{14} \mathrm{O}$; [M+H] Calculated: 211.1117; Found: 211.1118

M.P. $\left({ }^{\circ} \mathrm{C}\right): 67-68$

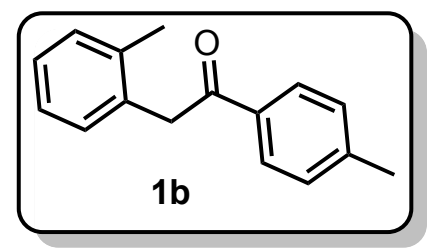

${ }^{1} \mathrm{H}$ NMR (400 MHz, $\left.\mathrm{CDCl}_{3}, \delta\right): 2.27\left(\mathrm{~s}, 3 \mathrm{H}, \mathrm{CH}_{3}\right), 2.43\left(\mathrm{~s}, 3 \mathrm{H}, \mathrm{CH}_{3}\right)$, $4.29\left(\mathrm{~s}, 2 \mathrm{H}, \mathrm{CH}_{2}\right), 7.12-7.22(\mathrm{~m}, 4 \mathrm{H}, \mathrm{Ar}-\mathrm{H}), 7.27-7.29$ (virtual d, J $=8 \mathrm{~Hz}, 2 \mathrm{H}, \mathrm{Ar}-\mathrm{H}$ ), $7.93-7.95$ (virtual $\mathrm{d}, \mathrm{J}=8 \mathrm{~Hz}, 2 \mathrm{H}, \mathrm{Ar}-\mathrm{H}$ ).

${ }^{13} \mathrm{C}$ NMR $\left(100 \mathrm{MHz}, \mathrm{CDCl}_{3}, \delta\right): 19.8,21.7,43.4,126.1,127.7,128.5$, 129.3, 130.3, 130.4, 133.7, 134.5, 136.9, 143.9, 197.1.

ATR-FTIR: 2901.09, 1680.96, 1605.98, 1336.15, 1221.83, 1202.00, 1173.36, 1000.86, 814.10 $\mathrm{cm}^{-1}$

HRMS: $\mathrm{C}_{16} \mathrm{H}_{16} \mathrm{O}$; [M+H] Calculated: 225.1273; Found: 225.1277 M.P. $\left({ }^{\circ} \mathrm{C}\right): 85.3-88$

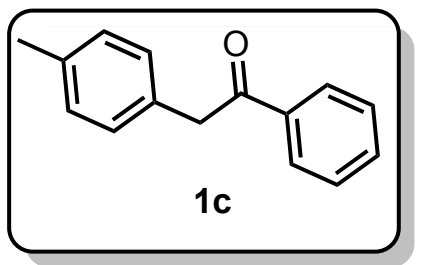

${ }^{1} \mathrm{H}$ NMR (400 MHz, $\left.\mathrm{CDCl}_{3}, \delta\right): 2.33$ (s, 3H, $\left.\mathrm{CH}_{3}\right), 4.25\left(\mathrm{~s}, 2 \mathrm{H}, \mathrm{CH}_{2}\right.$ ), 7.15-7.21 (two overlapped doublets, $J=8.4 \mathrm{~Hz}, 4 \mathrm{H}, p$-tolyl), 7.47$7.52(\mathrm{t}, J=7.6 \& 7.2 \mathrm{~Hz}, 1 \mathrm{H}, \mathrm{Ar}-\mathrm{H}), 7.56-7.62(\mathrm{t}, J=7.2 \mathrm{~Hz}, 2 \mathrm{H}$, Ar$\mathrm{H}), 8.03-8.08(\mathrm{~d}, J=7.2 \mathrm{~Hz}, 2 \mathrm{H}, \mathrm{Ar}-\mathrm{H})$.

${ }^{13} \mathrm{C}$ NMR $\left(100 \mathrm{MHz}, \mathrm{CDCl}_{3}, \delta\right): 20.9,40.0,128.5,129.2,129.3,131.3,133.0,136.4,197.7$.

ATR-FTIR: 2899, 1686.14, 1592.63, 1515.79, 1447.96, 1334.08, 1221.20, 1209.44, 994.94, $779.59,757.65,691.37 \mathrm{~cm}^{-1}$

HRMS: $\mathrm{C}_{15} \mathrm{H}_{14} \mathrm{O}$; [M+H] Calculated: 211.1117; Found: 211.1119

M.P. $\left({ }^{\circ} \mathrm{C}\right): 95-97$ 


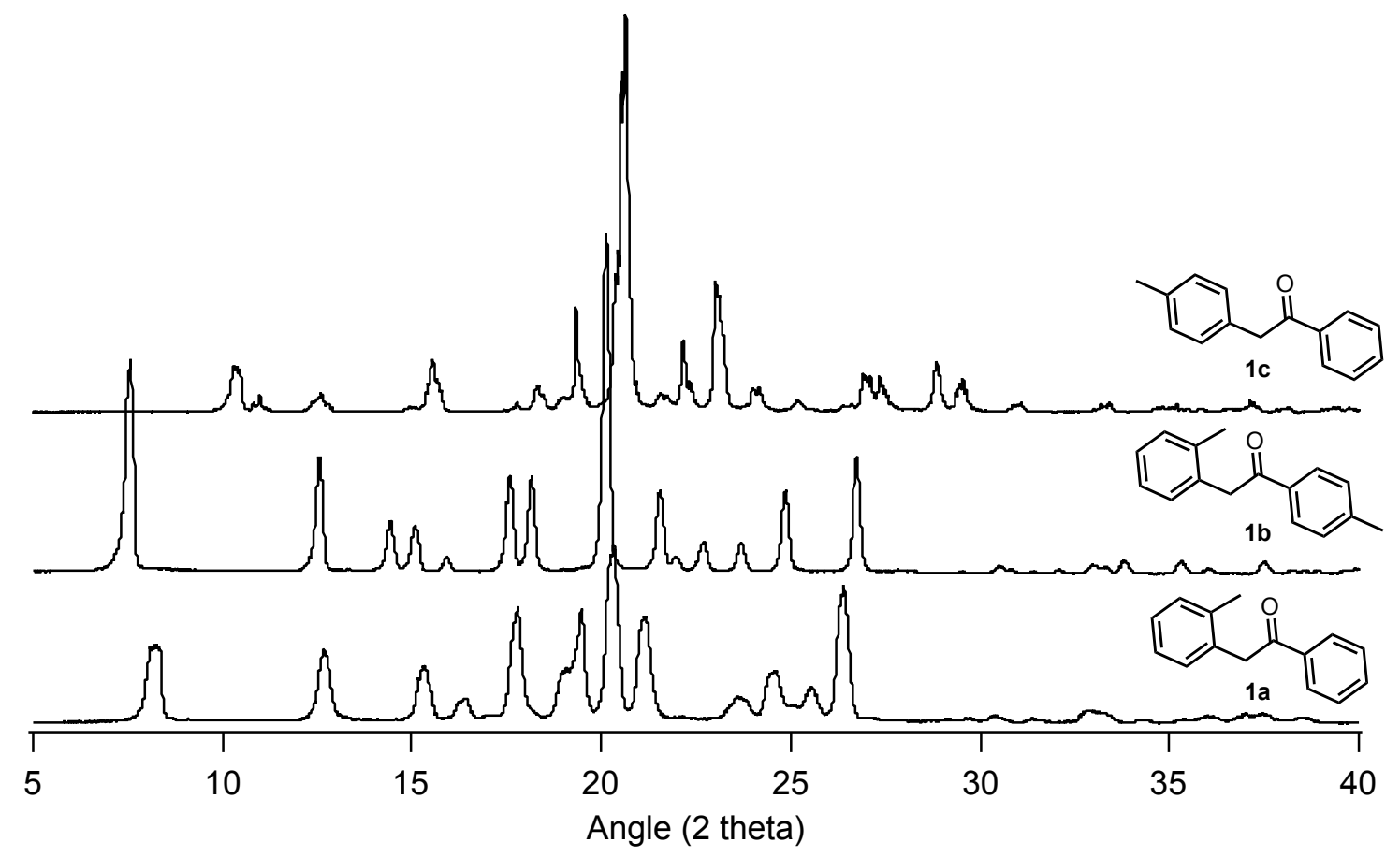

Figure S5. X-ray diffraction patterns of ketone 1a-c as bulk powder.

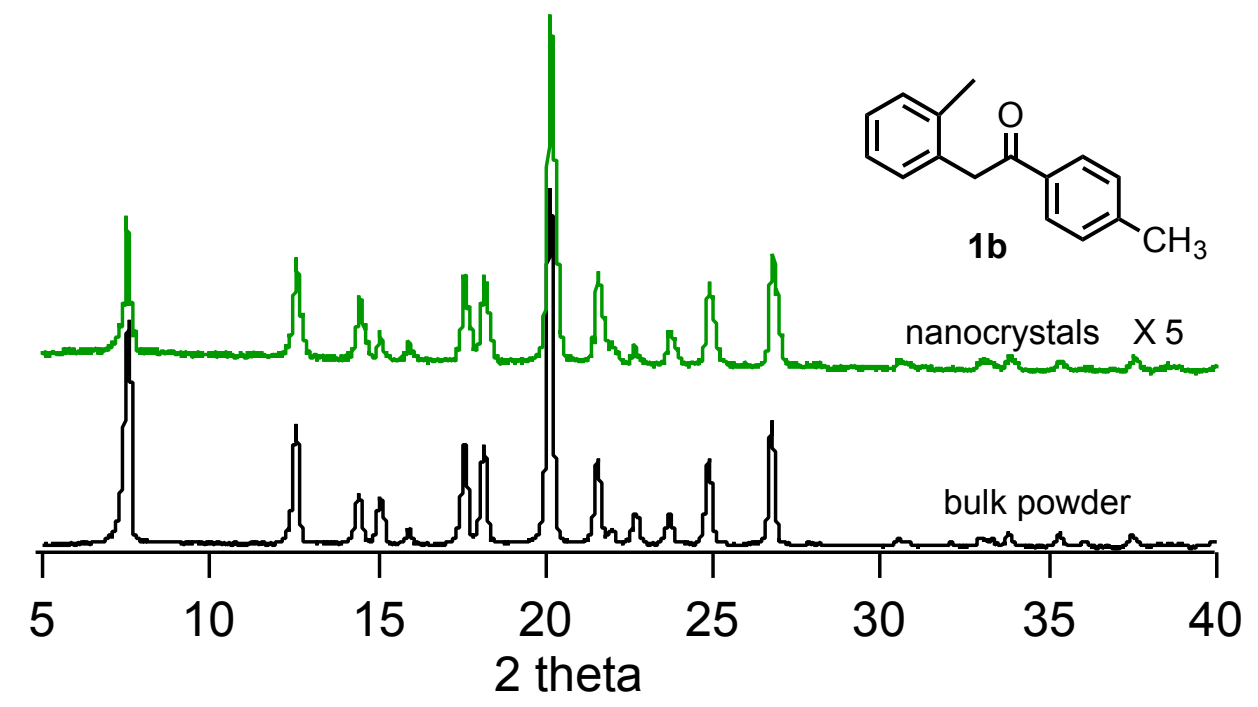

Figure S6. X-ray diffraction patterns of ketone $\mathbf{1 b}$ as bulk powder and nanocrystals (collected through centrifugation of the crystalline suspension). 
GENERAL IRRADIATION PROCEDURES AND CHARACTERIZATION OF PHOTOPRODUCTS $\mathbf{2 b - f}$

Solution phase irradiation

Benzene solutions of ketones 1a,b (8.5-13.5 mM) were purged with Argon for 30 min and photolyzed using medium pressure Hg lamp (450 W) with Pyrex UV cutoff (ca. $295 \mathrm{~nm}$ ) for $24 \mathrm{~h}$ at room temperature. After irradiation, the solutions were concentrated and the expected product(s) were isolated by flash chromatography on silica gel with $8-10 \%$ EtOAc:Hexanes solvent system; Isolated yields were $\mathbf{9 1 \%}$ and $80 \%$ for $\mathbf{2 a}$ and $\mathbf{2 b}$ respectively.

Photoproduct $\mathbf{2 a}$ has a low melting point at room temperature and thus is a viscous liquid whereas $\mathbf{2} \mathbf{b}$ is a crystalline solid compound.

Photoreaction in the crystals

Crystalline ketones 1a,b (10 mg) were grinded and sandwiched between two Pyrex microscope slides and irradiated using medium pressure $\mathrm{Hg}$ lamp (450 W) at room temperature for $24 \mathrm{~h}$ (at $25 \mathrm{~cm}$ from the lamp). TLC, GC and NMR analyses of the crude post-irradiated samples were performed and matched with authentic photoproducts or side products. Yields for isolated 2indanol photoproduct were calculated by ${ }^{1} \mathrm{H}$ NMR and GC: $\mathbf{2 a}(40 \%)$ and $\mathbf{2 b}(10 \%)$.

7c. Characterization of photoproduct $\mathbf{2 a , b}$

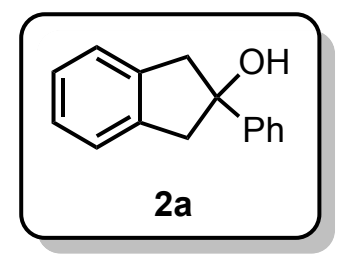

${ }^{1} \mathrm{H}$ NMR (400 MHz, $\left.\mathrm{CDCl}_{3}, \delta\right): 2.47$ (broad s, $\left.1 \mathrm{H}, \mathrm{OH}\right), 3.28-3.57$ (ABq, $\left.4 \mathrm{H}, \mathrm{CH}_{2}\right), 7.25-7.35$ (m, 5H, Ar-H), $7.39-7.43$ (virtual t, 2H, Ar-H), 7.60 -7.62 (virtual d, 2H, Ar-H).

${ }^{13} \mathrm{C}$ NMR $\left(100 \mathrm{MHz}, \mathrm{CDCl}_{3}, \delta\right): 49.2,83.4,125.1,125.3,126.9,127.3$, 128.4, 141.1, 145.7.

ATR-FTIR: 3389.91, 3024.35, 2910.35, 1602.45, 1482.54, 1459.48, 1447.10, 1209.26, 1039.47, $762.15,739.07,698.26 \mathrm{~cm}^{-1}$

HRMS: $\mathrm{C}_{15} \mathrm{H}_{14} \mathrm{O}$; [M] Calculated: 210.1039; Found: 210.0908

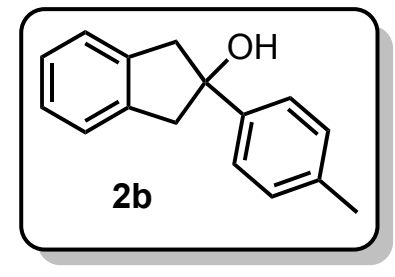

${ }^{1} \mathrm{H}$ NMR (400 MHz, $\left.\mathrm{CDCl}_{3}, \delta\right): 2.25(\mathrm{~s}, 1 \mathrm{H}, \mathrm{OH}), 2.42\left(\mathrm{~s}, 3 \mathrm{H}, \mathrm{CH}_{3}\right), 3.27$ - $3.55\left(\mathrm{ABq}, 4 \mathrm{H}, \mathrm{CH}_{2}\right.$ ), $7.22-7.33(\mathrm{~m}, 6 \mathrm{H}, \mathrm{Ar}-\mathrm{H}), 7.49-7.51$ (virtual d, $2 \mathrm{H}, \mathrm{Ar}-\mathrm{H})$.

${ }^{13} \mathrm{C}$ NMR (100 MHz, $\left.\mathrm{CDCl}_{3}, \delta\right): 21.1,49.2,83.3,125.1,125.3,126.9$ 
129.1, 136.9, 141.2, 142.9 .

ATR-FTIR: 3428.73, 1484.14, 1296.05, 1205.42, 1150.77, 1038, 821.48, 744.12, $722.32 \mathrm{~cm}^{-1}$

HRMS: $\mathrm{C}_{16} \mathrm{H}_{16} \mathrm{O}$; [M] Calculated: 224.1195; Found: 224.1152

M.P. $\left({ }^{\circ} \mathrm{C}\right): 80-83.7$

DYNAMIC LIGHT SCATTERING ANALYSIS OF CRYSTALLINE SUSPENSIONS OF 1a-c

The DLS data were recorded using a Beckman-Coulter N4 Plus particle analyzer with a $10 \mathrm{~mW}$ helium-neon laser at $632.8 \mathrm{~nm}$. The particle size was determined using the $62.6^{\circ}$ and $90^{\circ}$ detection angle and was calculated using the size distribution processor (SDP) analysis package provided by the manufacturer.

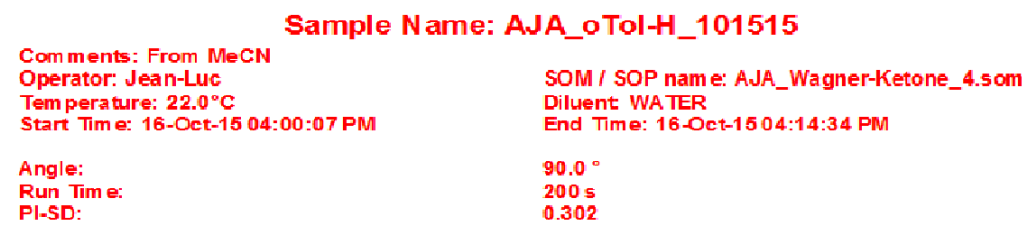

\begin{tabular}{|c|c|c|c|c|c|c|c|}
\hline \multicolumn{8}{|c|}{ Unimodal Results Summary } \\
\hline Reptlf. & $\begin{array}{l}\text { Mean } \\
\text { (nrm!) }\end{array}$ & $\begin{array}{c}\text { Sitd_Dev } \\
\text { (nmm) }\end{array}$ & $\begin{array}{l}\text { Biaseline } \\
\text { Error }\end{array}$ & P.t. & Counts's & $\begin{array}{c}\text { Diff.Coef } \\
\left(\mathrm{m}^{2} / \mathrm{s}\right)\end{array}$ & Overflow \\
\hline Rept.1 & 247.4 & 9018.4 & $0.01 \%$ & -0.415 & $1.31 e+05$ & $1.83 e-12$ & 0 \\
\hline Rept.2 & 231.2 & 101.8 & $0.00 \%$ & 0.433 & $1,32 e+05$ & $1.96=-12$ & 0 \\
\hline Rept.3 & 242.2 & 70.1 & $0.01 \%$ & -0.093 & $1.340+05$ & $1.870-12$ & 0 \\
\hline Awerage & 240.3 & 90.40 & & $-0,025$ & & & \\
\hline
\end{tabular}

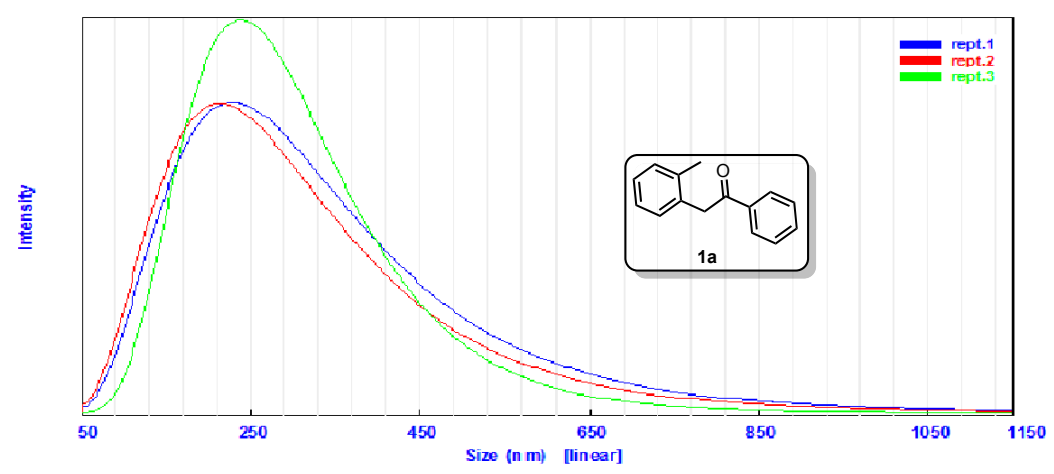

Figure S7: DLS results for 1a depicting average size $(240 \mathrm{~nm})$ for the nanocrystals dispersed in water. 


\begin{tabular}{|c|c|c|c|c|c|c|c|}
\hline \multicolumn{4}{|c|}{$\begin{array}{l}\text { Comments: From THF } \\
\text { Operator: Jean-Luc } \\
\text { Temperatu re: } 22.0^{\circ} \mathrm{C} \\
\text { Start Time: } 12 \text {-Oct-15 03:32:36 PM }\end{array}$} & \multicolumn{4}{|c|}{$\begin{array}{l}\text { SOM / SOP name: AJA_Wagner-Ketone_4.som } \\
\text { Diluent: WATER } \\
\text { End Time: } 12-0 \mathrm{ct}-15 \text { 03:47:03PM }\end{array}$} \\
\hline \multicolumn{3}{|l|}{$\begin{array}{l}\text { Angle: } \\
\text { Run Time: } \\
\text { PI-SD: }\end{array}$} & & \multicolumn{4}{|l|}{$\begin{array}{l}90.0^{\circ} \\
200 \mathrm{~s} \\
0.017\end{array}$} \\
\hline \multicolumn{8}{|c|}{ Unimodal Results S ummary } \\
\hline Rept= & $\begin{array}{l}\text { Mean } \\
\text { (nm) }\end{array}$ & $\begin{array}{c}\text { Std. Dev } \\
\text { (nm]) }\end{array}$ & $\begin{array}{c}\text { Baseine } \\
\text { Error }\end{array}$ & PI. & Counts/s & $\begin{array}{c}\text { DiffC. oef } \\
\left(\mathrm{m}^{2} / \mathrm{s}\right)\end{array}$ & Overflown \\
\hline Rept 1 & 394.4 & 169.8 & $0.00 \%$ & 0.359 & $4.15 e+05$ & $1.15 \mathrm{e}-12$ & 0 \\
\hline Rept2 & 384.9 & 168.2 & $0.01 \%$ & 0.405 & $4.15 e+05$ & $1.18 \mathrm{e}-12$ & 0 \\
\hline Rept 3 & 389.0 & 168.1 & $0.01 \%$ & 0.369 & $4.18 e+05$ & $1.16 \mathrm{e}-12$ & 0 \\
\hline Average & 389.4 & $168.70^{-}$ & & $0.37 \pi$ & & & \\
\hline
\end{tabular}

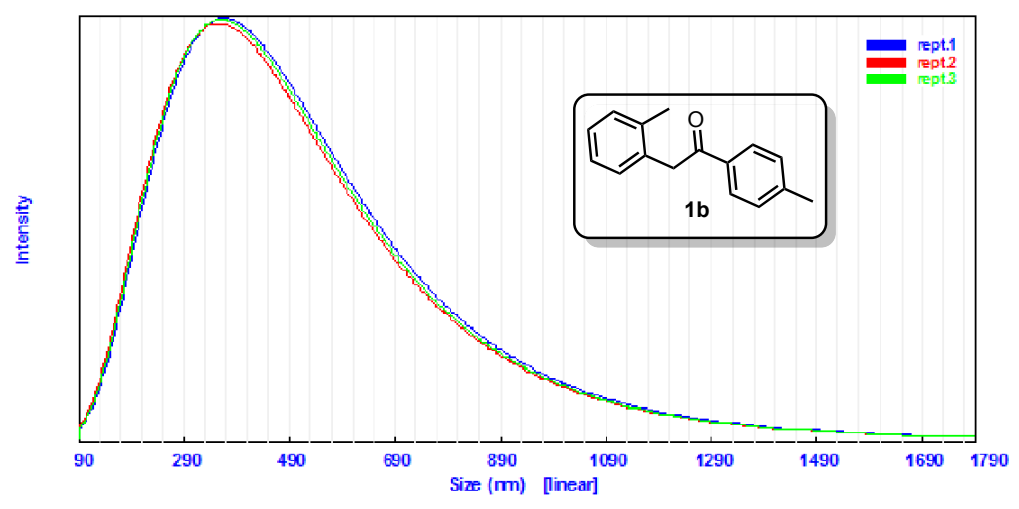

Figure S8: DLS results for $\mathbf{1 b}$ depicting average size $(389 \mathrm{~nm})$ for the nanocrystals dispersed in water. 
Sample Name: AJA2_109_pTol-H_tr1

Comments: pTol-H_tr1 in water Operator:

Temperature: $22.0^{\circ} \mathrm{C}$

Start Time: 02-Dec-14 12:35:39 PM

Angle:

Run Time:

PI-SD:
SOM / SOP name: AJA_Wagner-Ketone_2.som

Diluent: WATER

End Time: 02-Dec-14 01:00:07 PM

$62.6^{\circ}$

$400 \mathrm{~s}$

0.049

\begin{tabular}{|c|c|c|c|c|c|c|c|}
\hline \multicolumn{8}{|c|}{$\begin{array}{l}\text { modal Distribution Reprod } \\
\text { Unimodal Results Summary }\end{array}$} \\
\hline Rep\#. & $\begin{array}{l}\text { Mean } \\
(\mathrm{nm})\end{array}$ & $\begin{array}{l}\text { Std.Dev } \\
(\mathrm{nm})\end{array}$ & $\begin{array}{c}\text { Baseline } \\
\text { Error }\end{array}$ & P.I. & Counts/s & $\begin{array}{c}\text { Diff.Coef } \\
\left(\mathrm{m}^{2} / \mathrm{s}\right)\end{array}$ & Overflow \\
\hline Rept.1 & 3994.1 & 1791.8 & $5.07 \%$ & 0.516 & $6.87 \mathrm{e}+05$ & $1.13 \mathrm{e}-13$ & 0 \\
\hline Rept.2 & 4763.6 & 2178.0 & $3.13 \%$ & 0.638 & $6.38 e+05$ & $9.50 \mathrm{e}-14$ & 0 \\
\hline Rept.3 & 4124.9 & 1852.7 & $0.92 \%$ & 0.523 & $5.91 \mathrm{e}+05$ & $1.10 \mathrm{e}-13$ & 0 \\
\hline Average & 4294.2 & 1940.85 & & 0.559 & & & \\
\hline
\end{tabular}

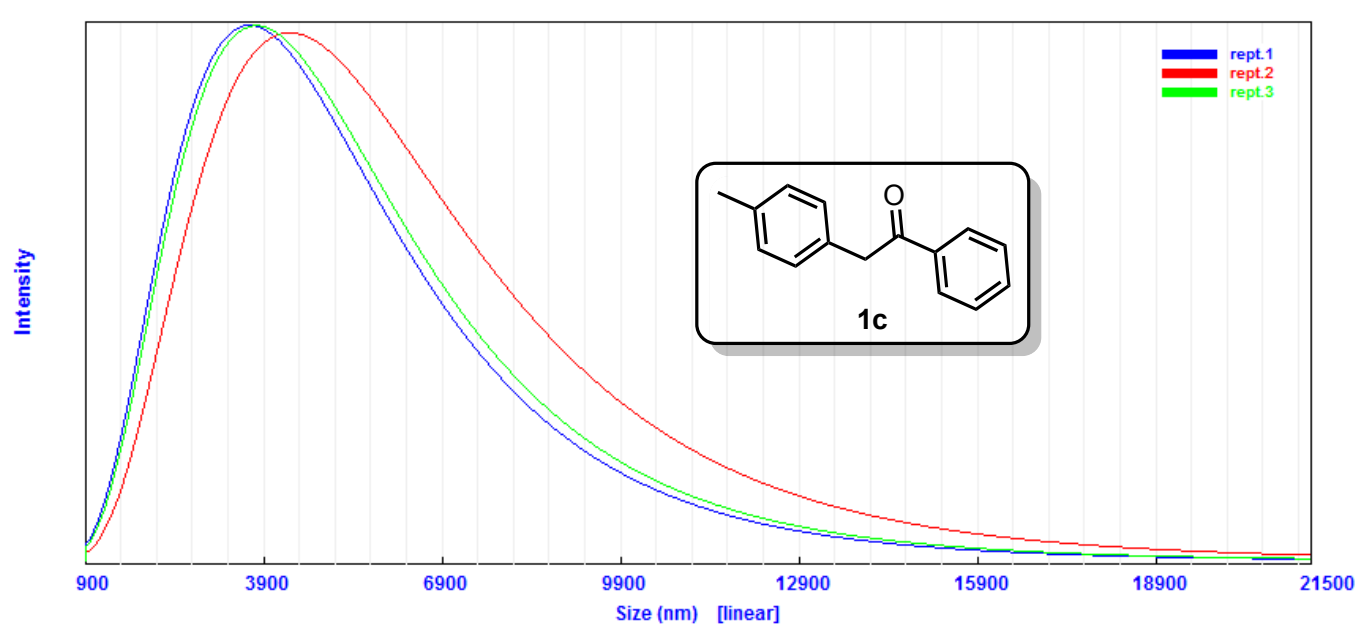

Figure S9: DLS results for 1c depicting micron size crystalline particles dispersed in water. Detection angles $62.6^{\circ}$ and $90^{\circ}$ gave the same result. The average particle size in the case of 1c was consistent when samples from from different preparations were measured. Despite the large DLS particle sizes, steady state and transient optical spectra were of good quality. 


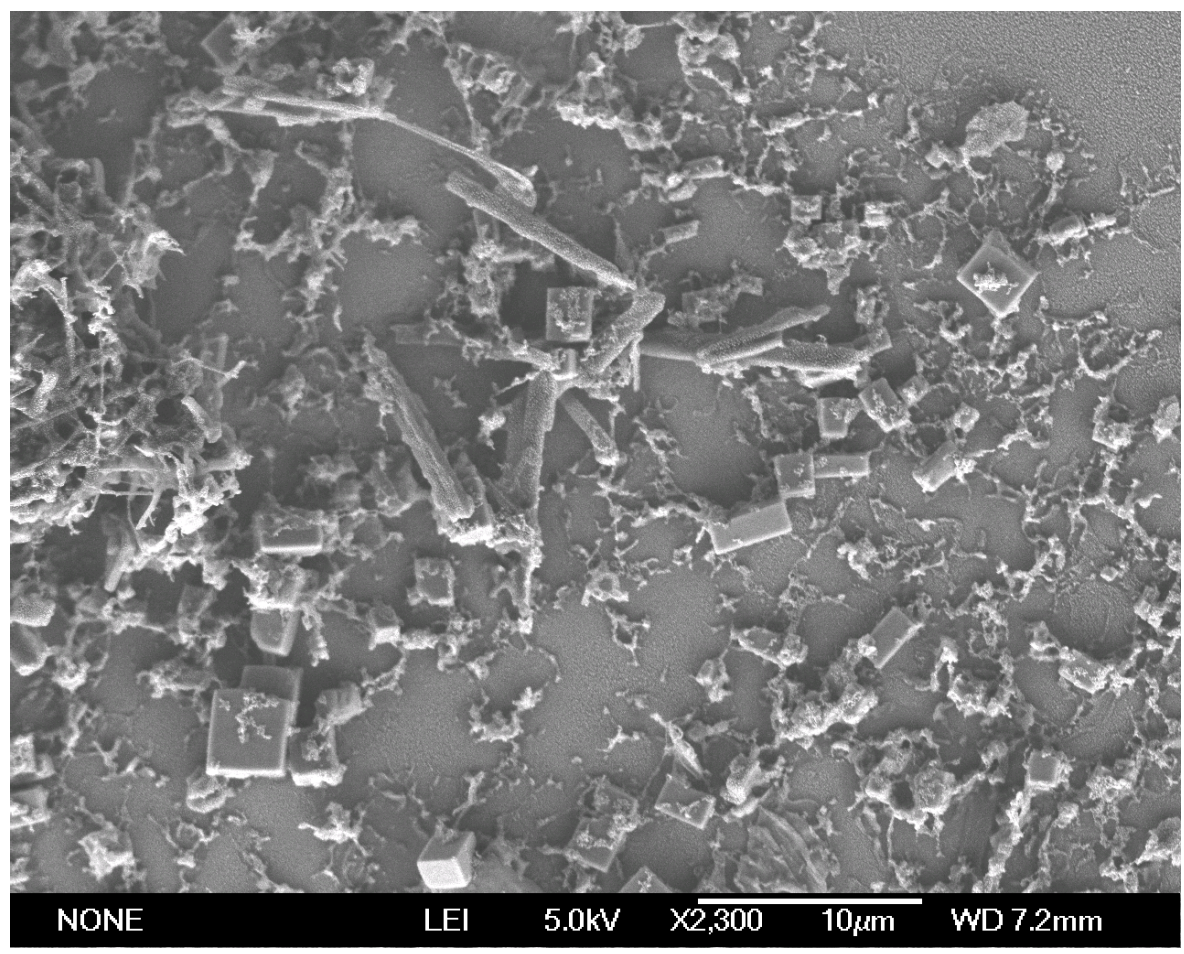

Figure S10: SEM images of a sample preparations using ketone 1c showing crystals with dimensions in the ca. $1 \mu \mathrm{m}$ range $\mathrm{In}$ their smaller dimension. As amorphous precipitate is formed by aggregation of CTAB. 
${ }^{1} \mathrm{H}$ NMR $\left(400 \mathrm{MHz}, \mathrm{CDCl}_{3}\right)$

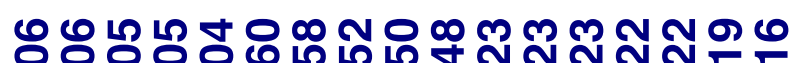

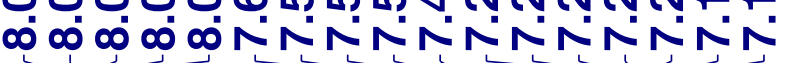

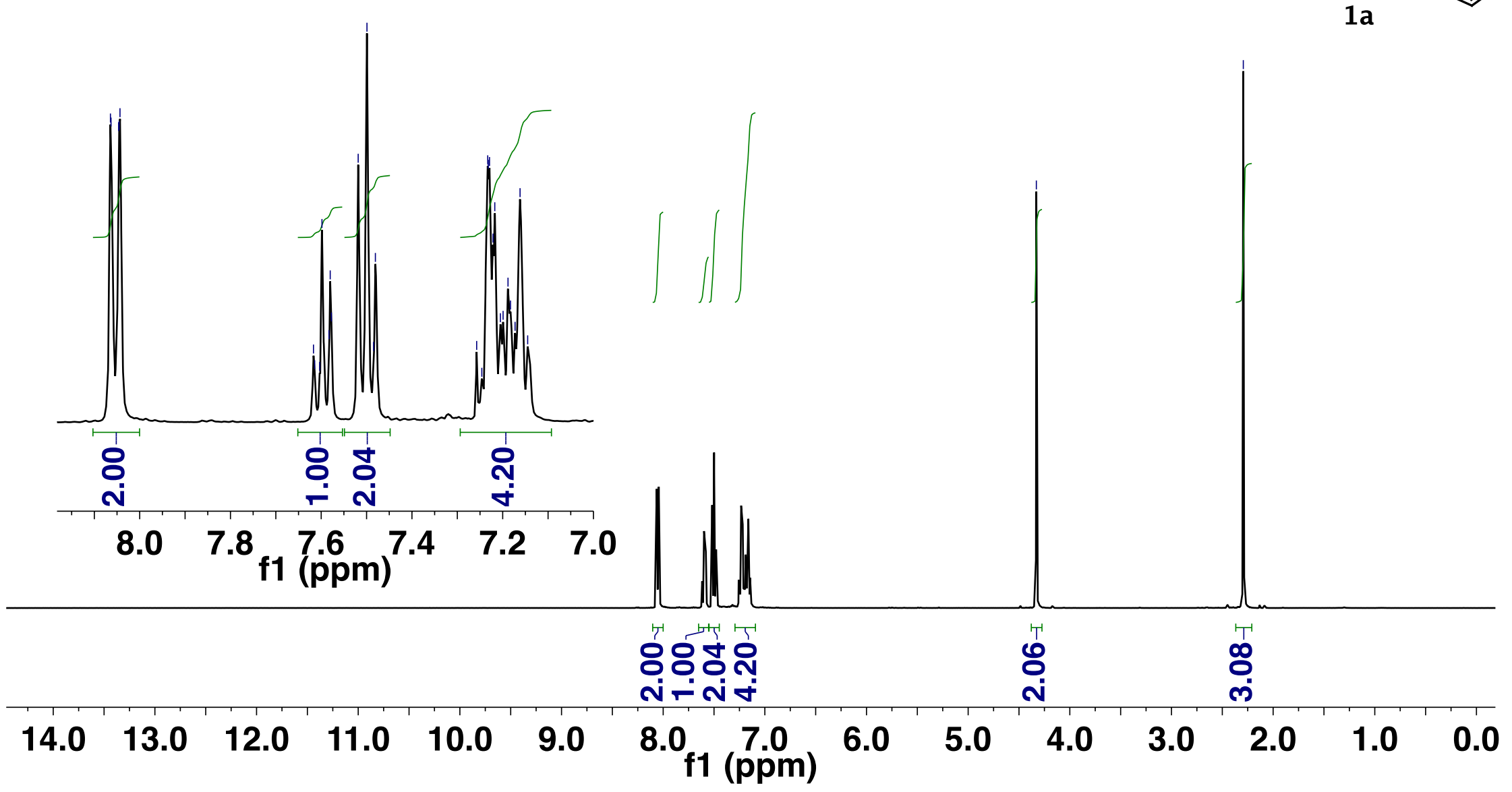

m

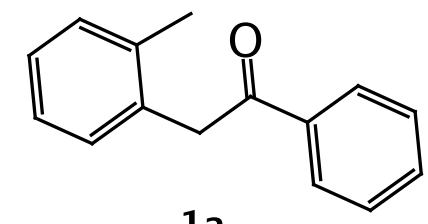

Figure S11: ${ }^{1} \mathrm{H}$ NMR spectrum of $1 \mathrm{a}$ in $\mathrm{CDCl}_{3}$. 


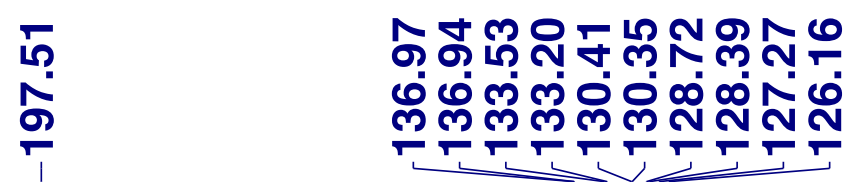

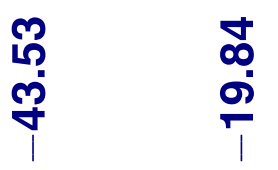

${ }^{13} \mathrm{C}$ NMR $\left(100 \mathrm{MHz}_{1} \mathrm{CDCl}_{3}\right)$
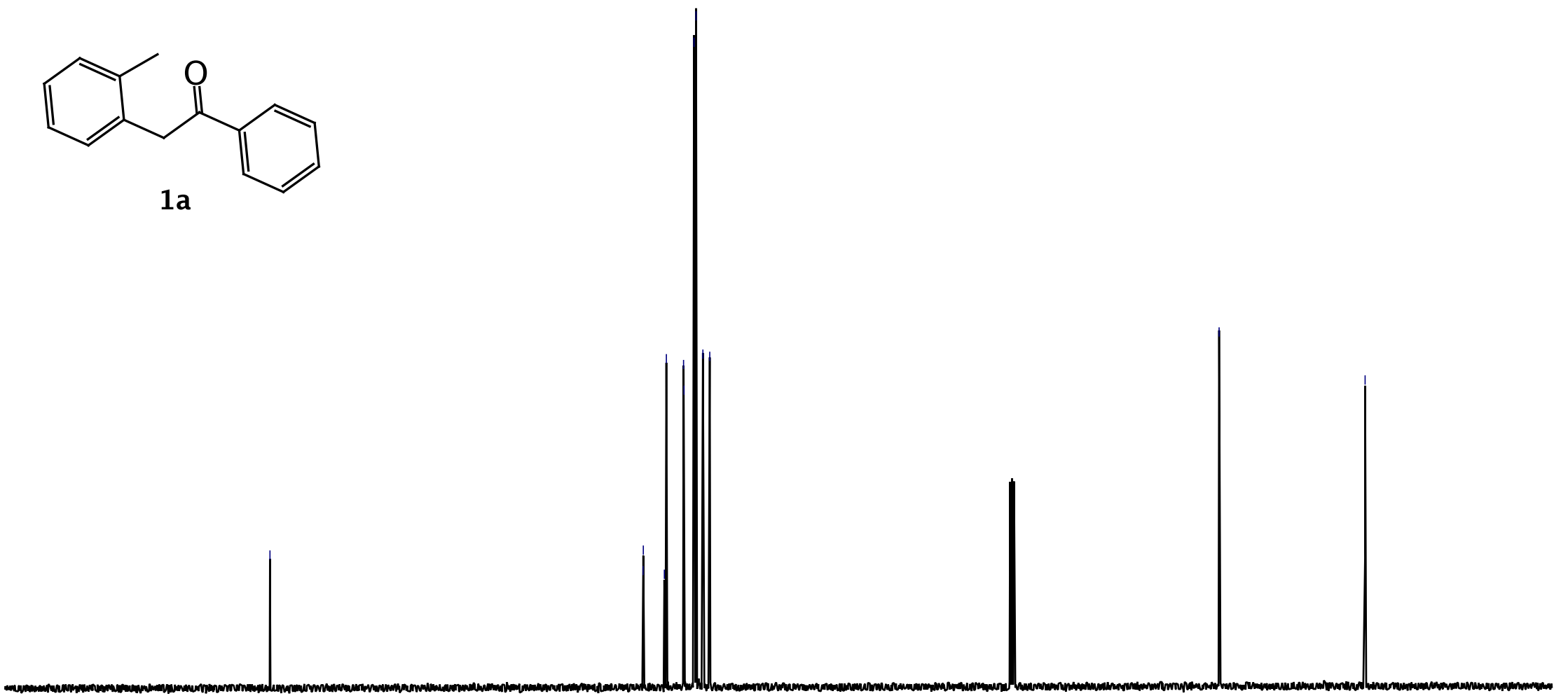

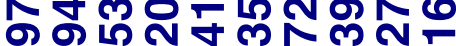

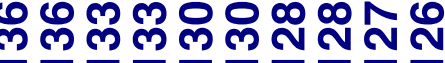

$\begin{array}{lllllllllllll}230 & 210 & 190 & 170 & 150 & 130_{\mathrm{f} 1(\mathrm{ppm})} & 90 & 70 & 50 & 30 & 10 & -1 \mathrm{C}\end{array}$

Figure S12: ${ }^{13} \mathrm{C}$ NMR spectrum of $1 \mathrm{a}$ in $\mathrm{CDCl}_{3}$. 
${ }^{1} \mathrm{H}$ NMR $\left(400 \mathrm{MHz}, \mathrm{CDCl}_{3}\right)$

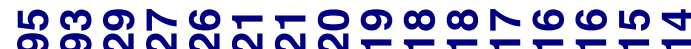
NNNNNNNNNNNNNN

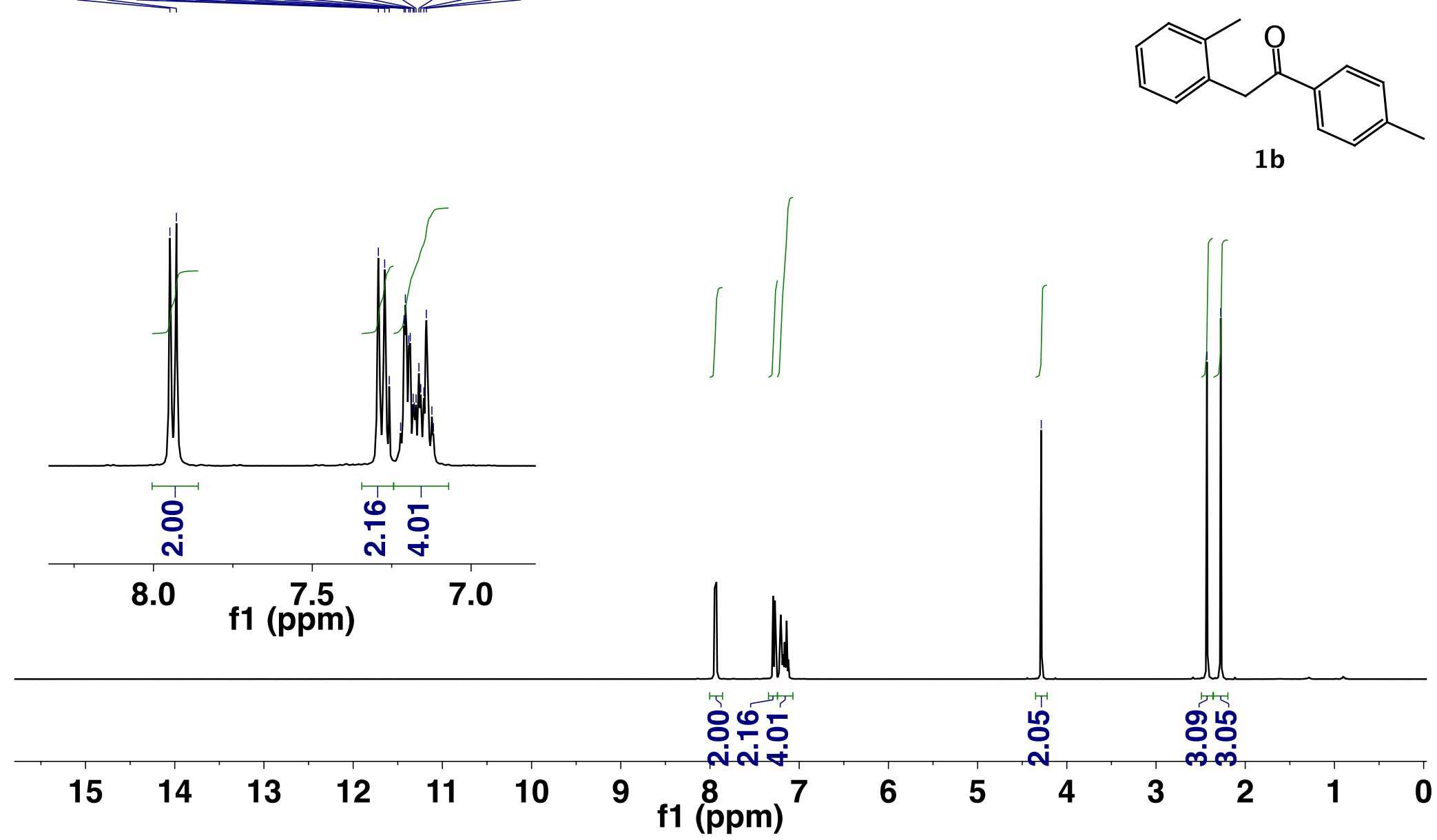

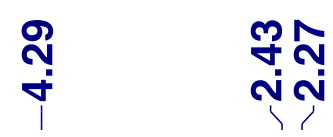

Figure S13: ${ }^{1} \mathrm{H}$ NMR spectrum of $1 \mathrm{~b}$ in $\mathrm{CDCl}_{3}$. 


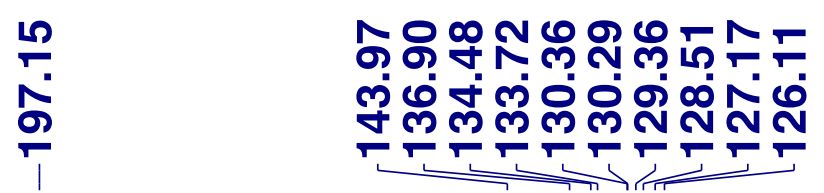

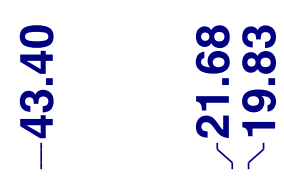

${ }^{13} \mathrm{C} \mathrm{NMR}\left(100 \mathrm{MHz}, \mathrm{CDCl}_{3}\right)$

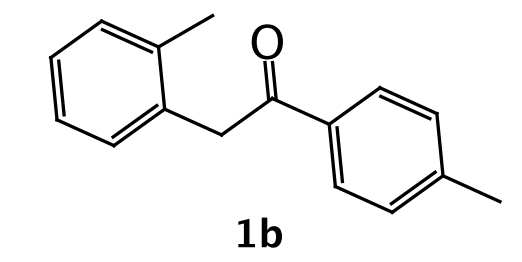

$1 b$

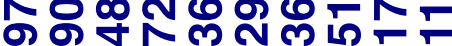

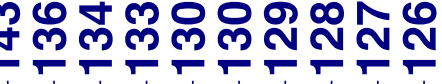

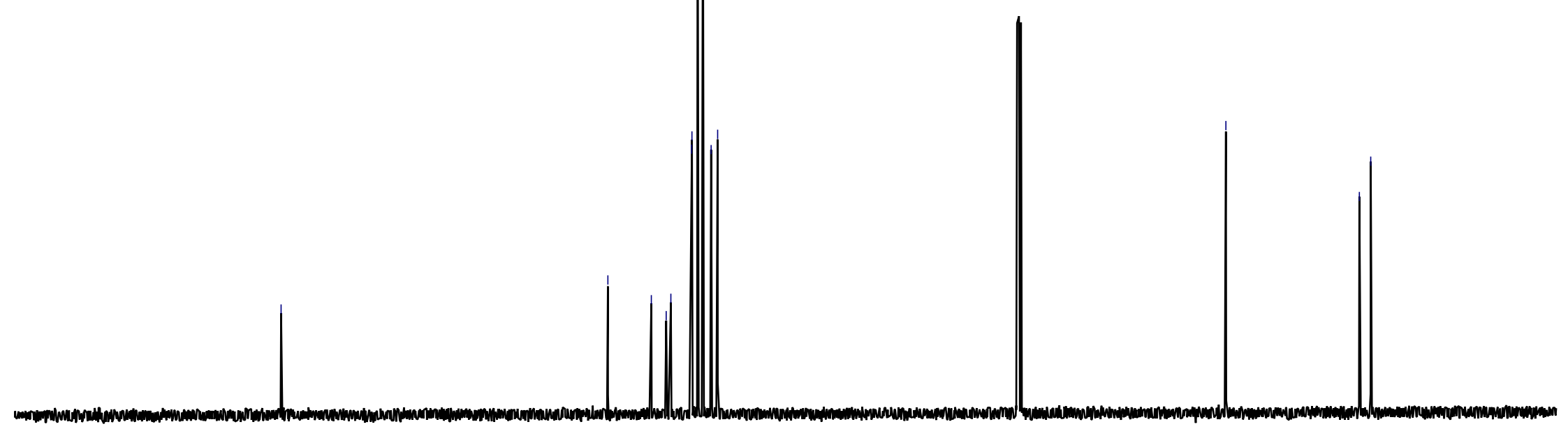

$\begin{array}{llllllllllll}230 & 210 & 190 & 170 & 150 & \begin{array}{c}130_{\mathrm{f} 1}(\mathrm{ppm}) \\ 110\end{array} & 90 & 70 & 50 & 30 & 10 & -1 \mathrm{C}\end{array}$

Figure S14: ${ }^{13} \mathrm{C}$ NMR spectrum of $1 \mathrm{~b}$ in $\mathrm{CDCl}_{3}$. 
${ }^{1} \mathrm{H}$ NMR $\left(400 \mathrm{MHz}, \mathrm{CDCl}_{3}\right)$

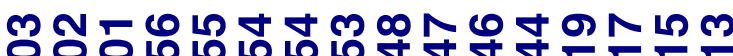

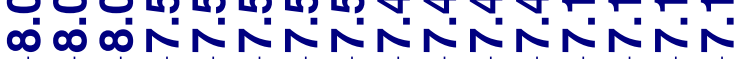

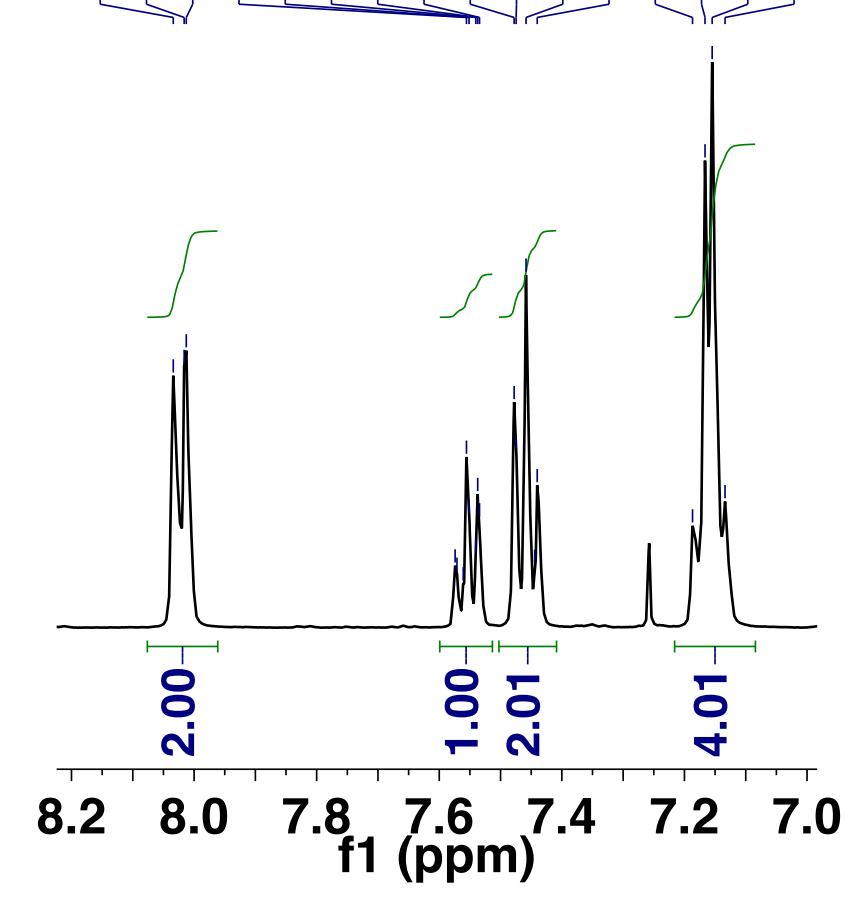

ஸั

m

N

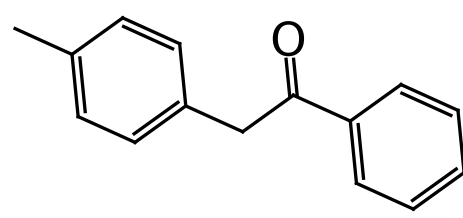

1c

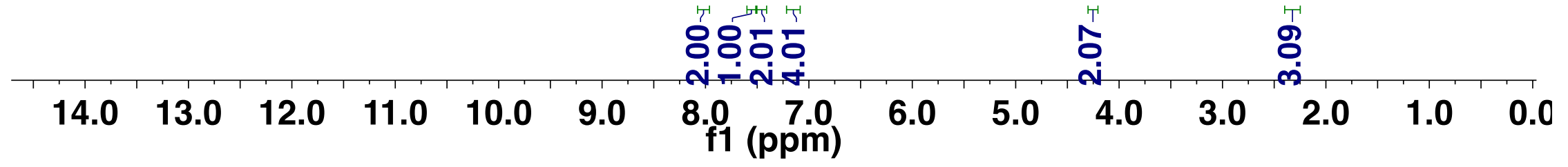

Figure S15: ${ }^{1} \mathrm{H}$ NMR spectrum of $1 \mathrm{c}$ in $\mathrm{CDCl}_{3}$. 


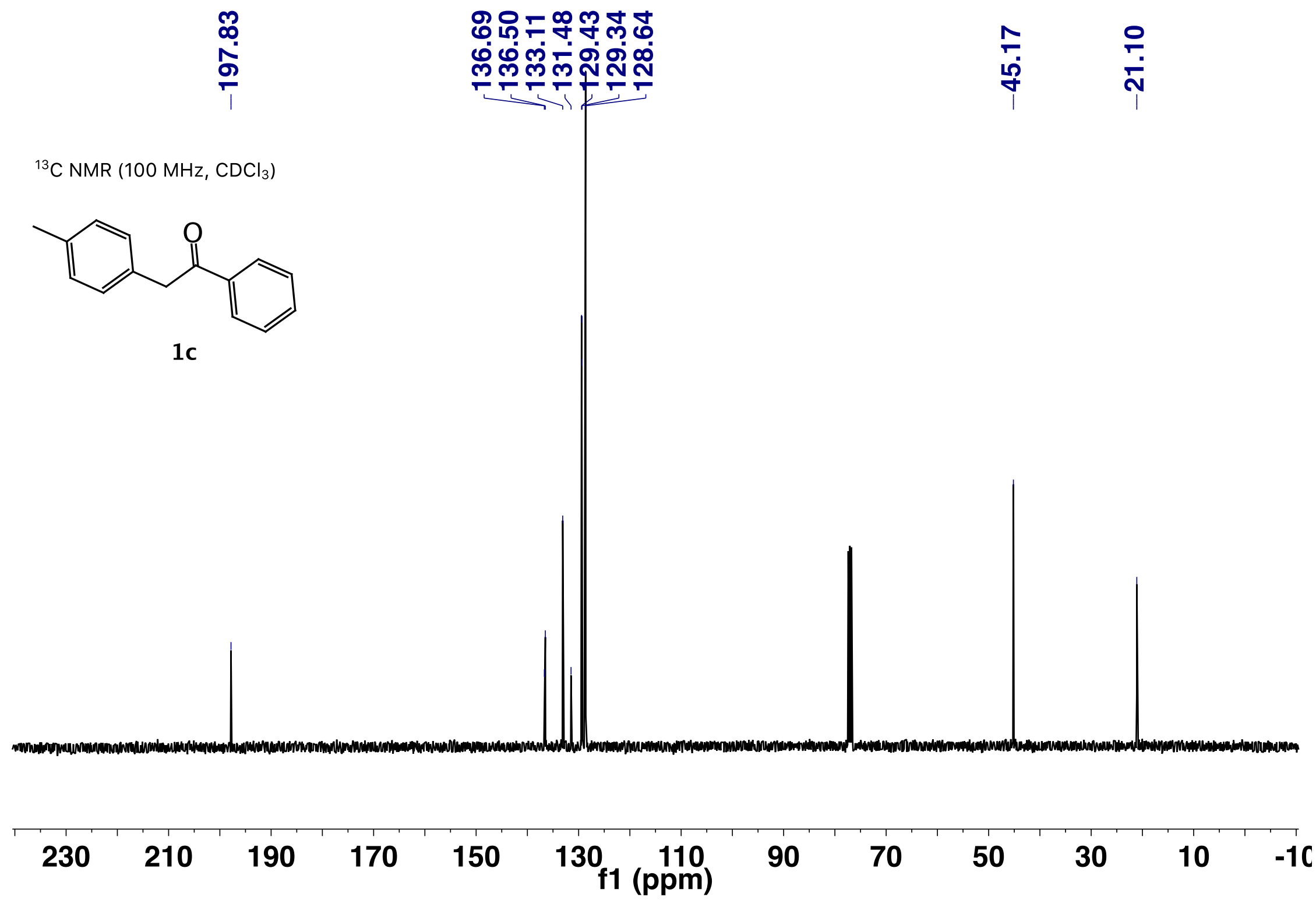

Figure S16: ${ }^{13} \mathrm{C}$ NMR spectrum of $1 \mathrm{c}$ in $\mathrm{CDCl}_{3}$. 
${ }^{1} \mathrm{H}$ NMR $\left(400 \mathrm{MHz}, \mathrm{CDCl}_{3}\right)$

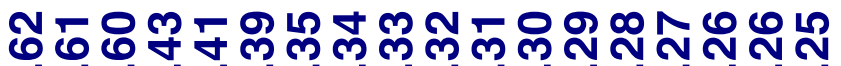

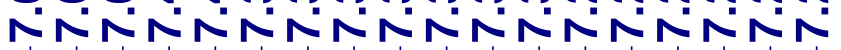

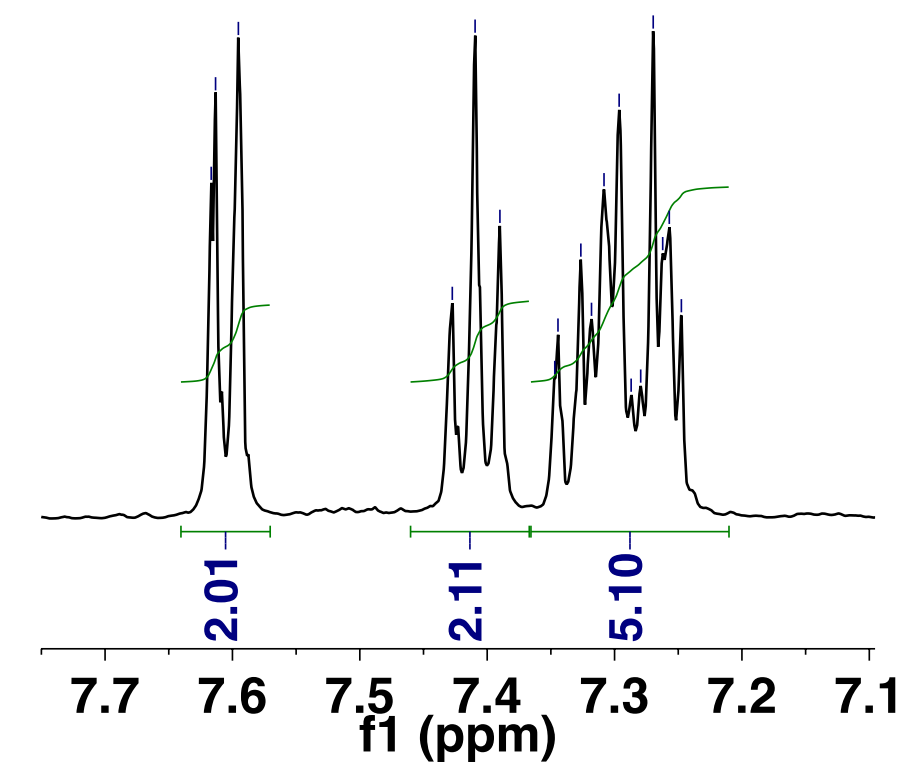

กิ กิ ำ

ल लं ल

if

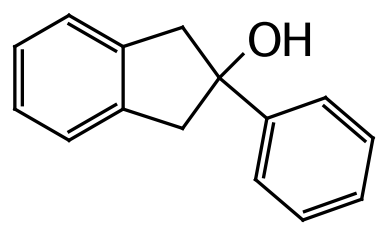

$2 a$

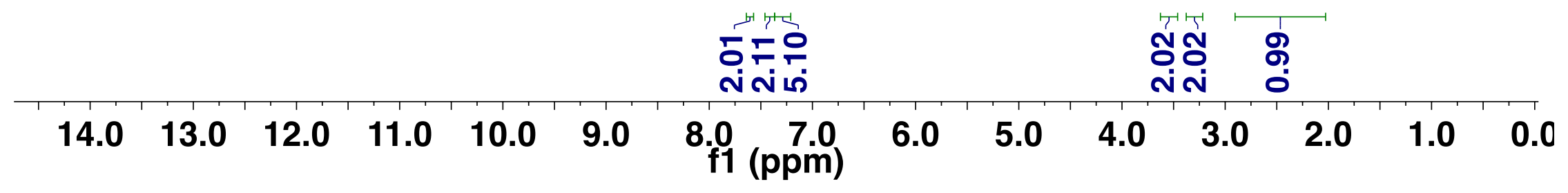

Figure S17: ${ }^{1} \mathrm{H}$ NMR spectrum of $2 \mathrm{a}$ in $\mathrm{CDCl}_{3}$. 


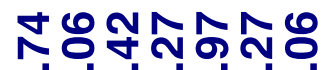

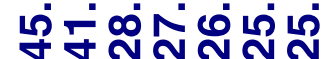

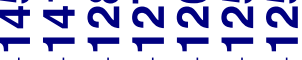

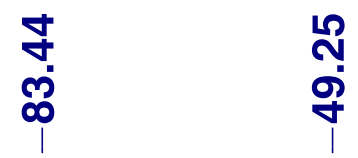

${ }^{13} \mathrm{C}$ NMR $\left(100 \mathrm{MHz}, \mathrm{CDCl}_{3}\right)$

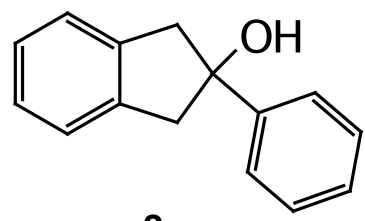

$2 a$

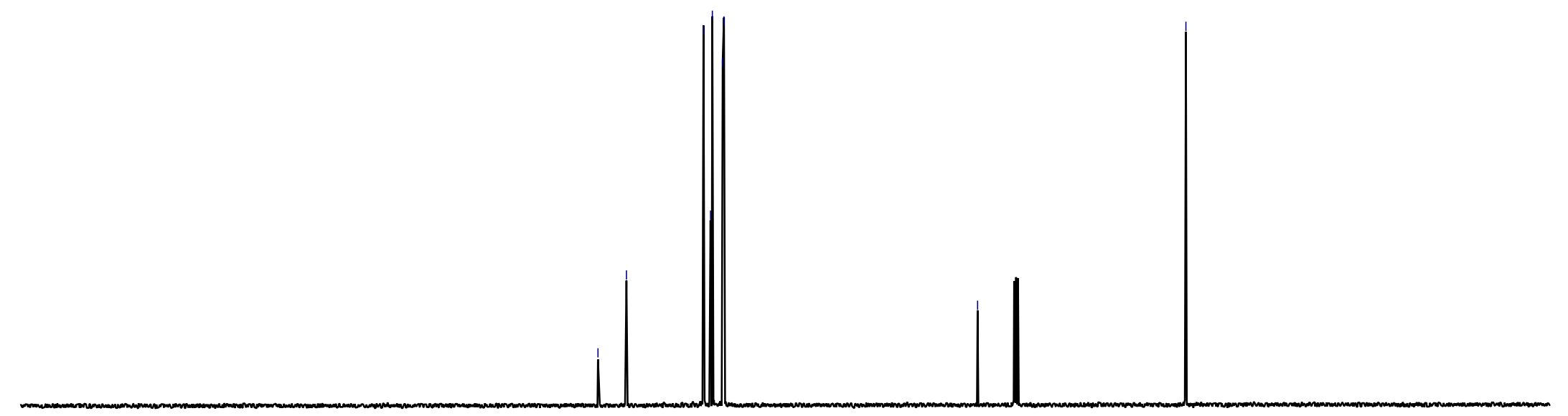

\section{0 \\ 210 \\ 190 \\ 170 \\ 150 \\ 130 \\ 110 \\ 90}

Figure S18: ${ }^{13} \mathrm{C}$ NMR spectrum of $2 \mathrm{a}$ in $\mathrm{CDCl}_{3}$. 


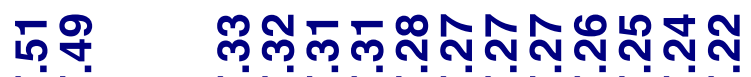

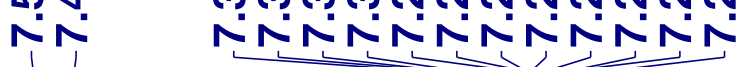
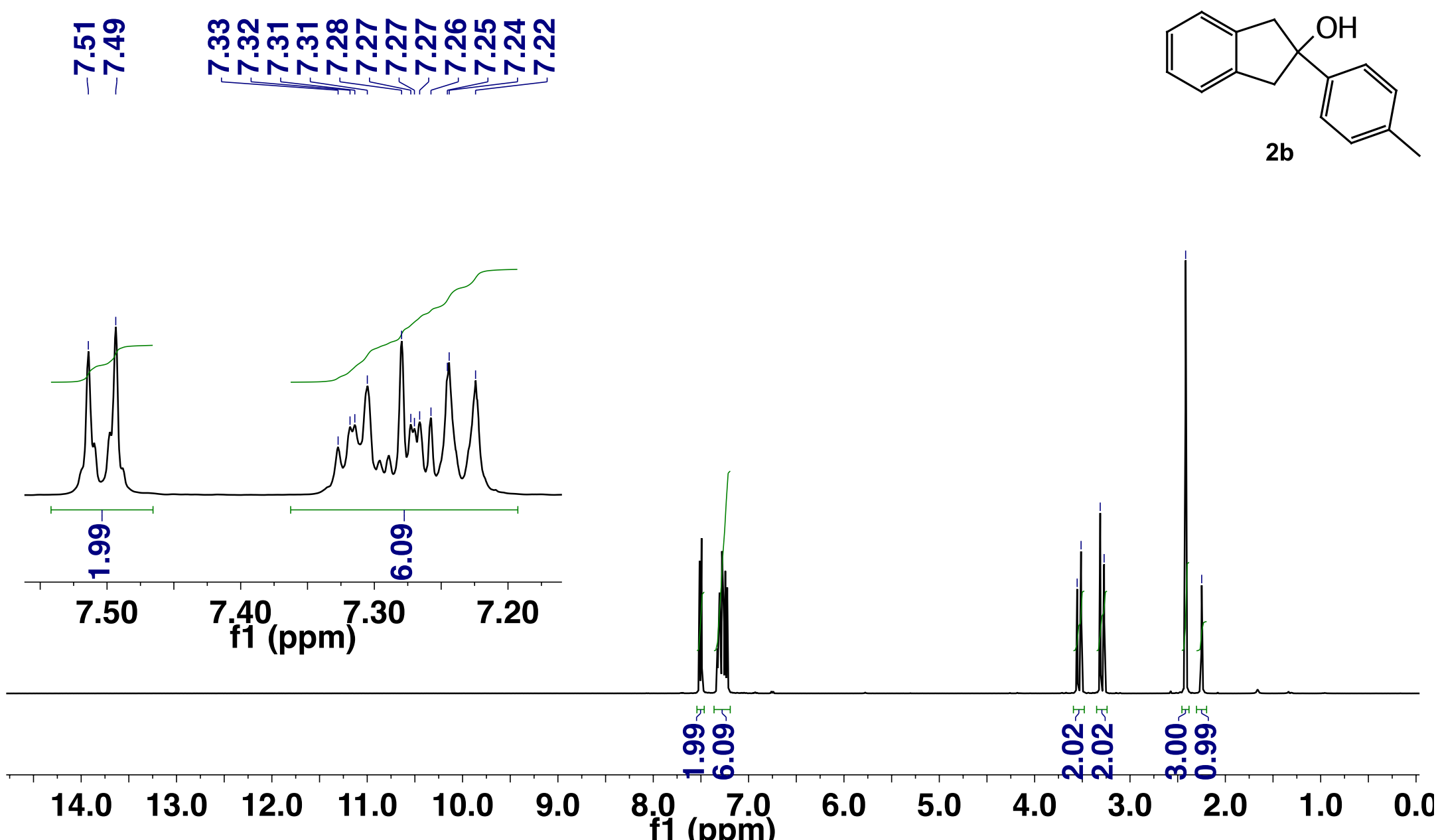

Figure S19: ${ }^{1} \mathrm{H}$ NMR spectrum of $\mathbf{2 b}$ in $\mathrm{CDCl}_{3}$. 


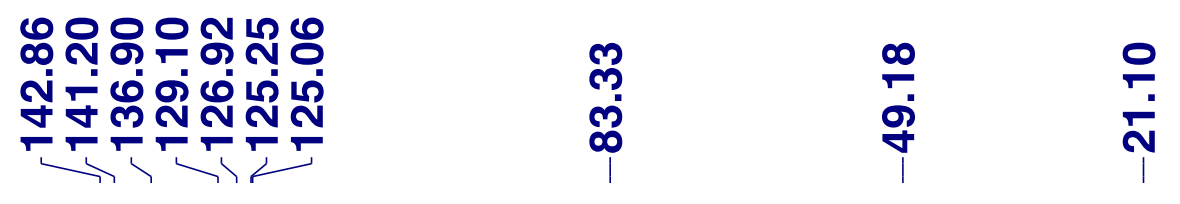

${ }^{13} \mathrm{C} \mathrm{NMR}\left(100 \mathrm{MHz}, \mathrm{CDCl}_{3}\right)$
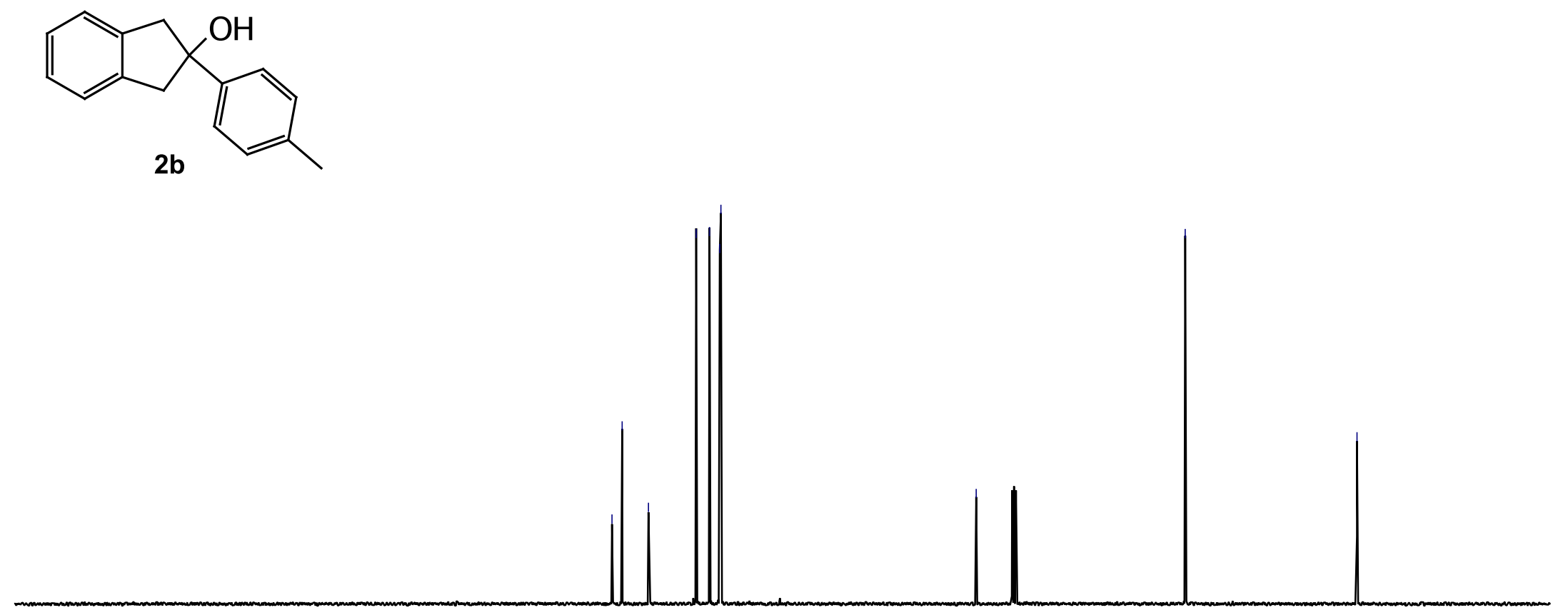

\begin{tabular}{|c|c|c|c|c|c|c|c|c|c|c|c|}
\hline 230 & 210 & 190 & 170 & 150 & 130 & $\begin{array}{c}110 \\
\text { ppm) }\end{array}$ & 90 & 70 & 50 & 30 & 10 \\
\hline
\end{tabular}

Figure S20: ${ }^{13} \mathrm{C}$ NMR spectrum of $\mathbf{2 b}$ in $\mathrm{CDCl}_{3}$. 


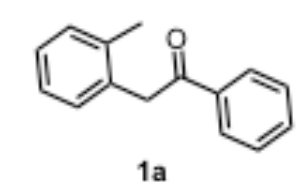

$1 \mathrm{a}$

Date: Wednesday, April 15, 2015

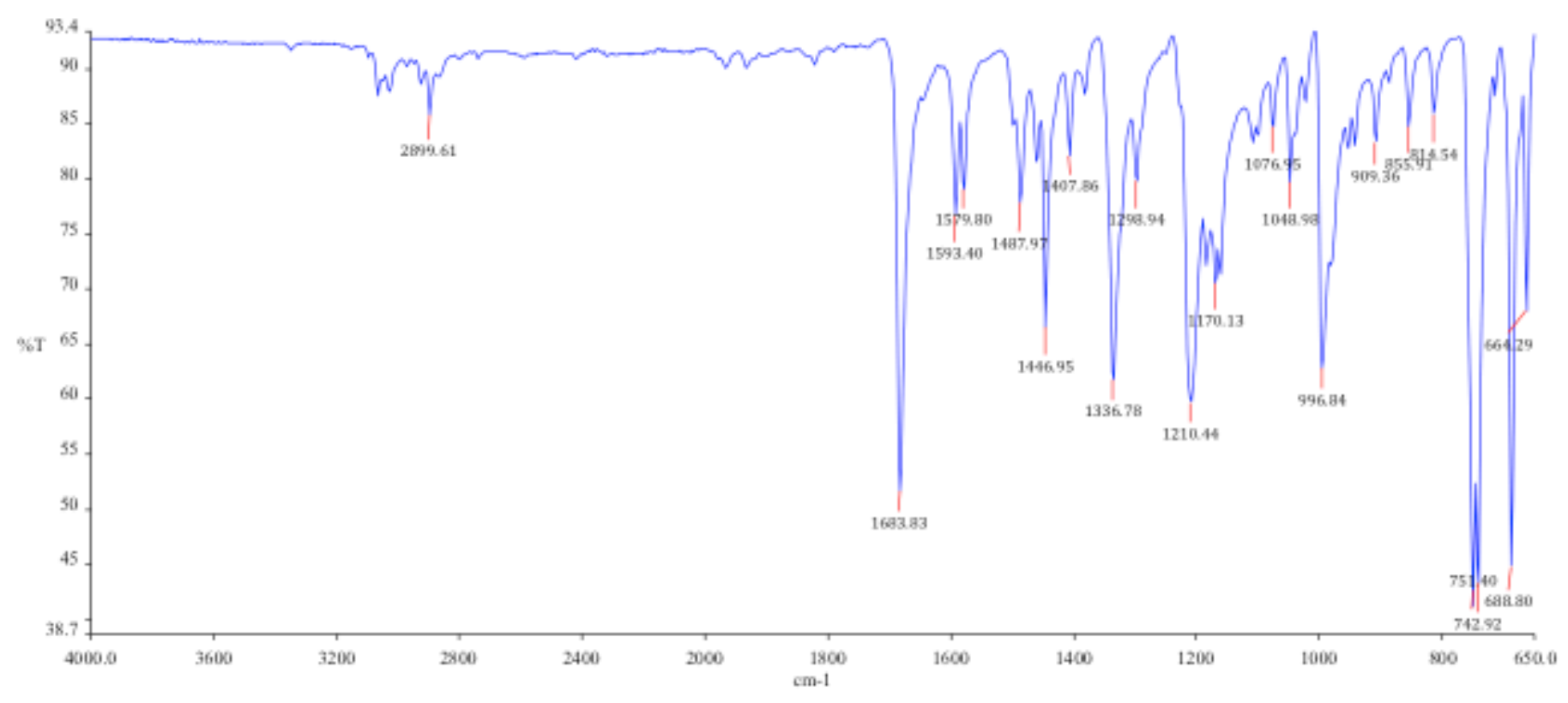

Figure S21: IR spectrum of 1a. 


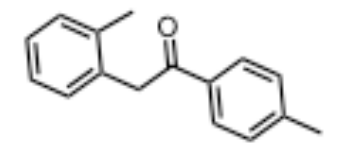

$1 b$

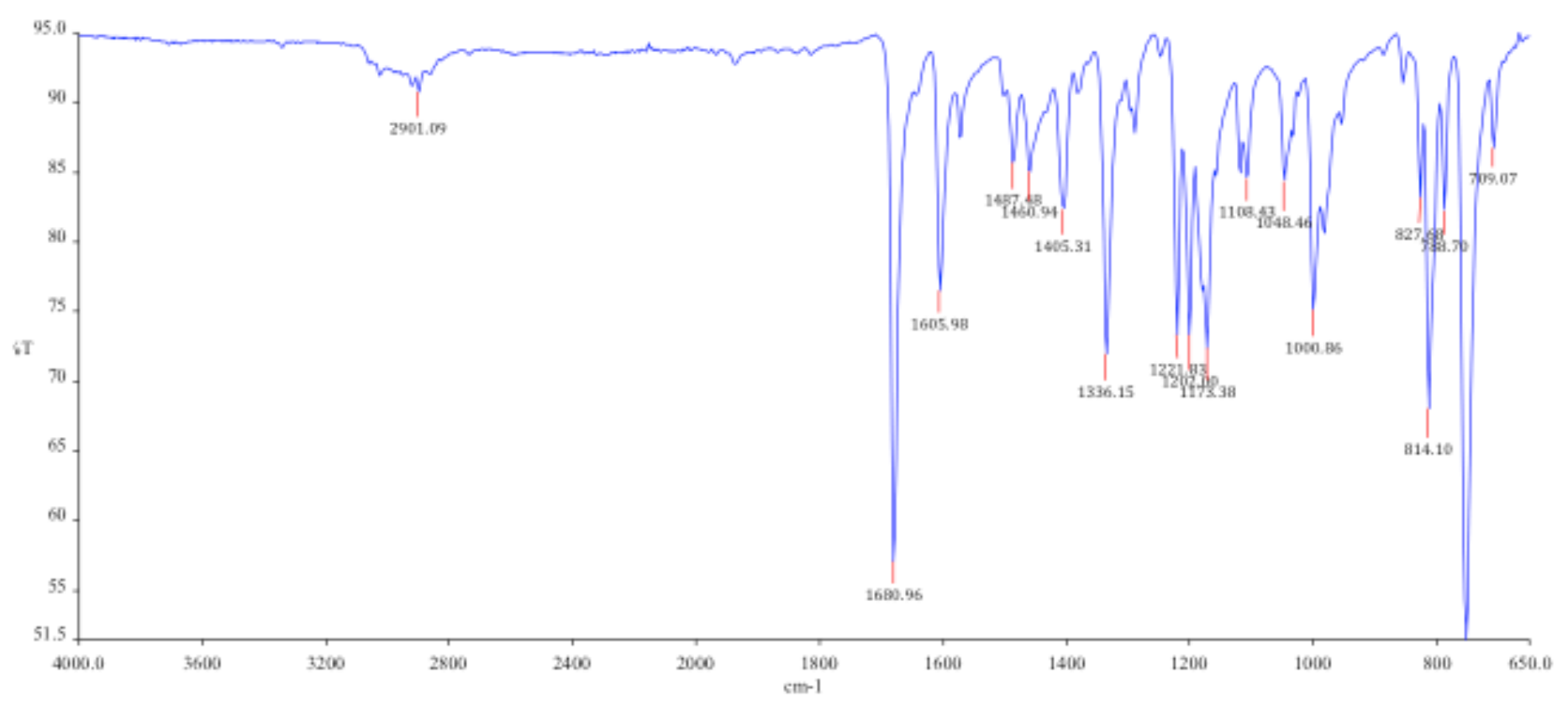

Figure S22: IR spectrum of $\mathbf{1 b}$. 


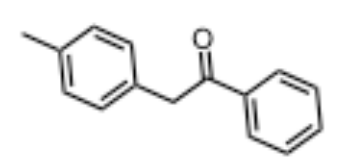

$1 \mathrm{c}$

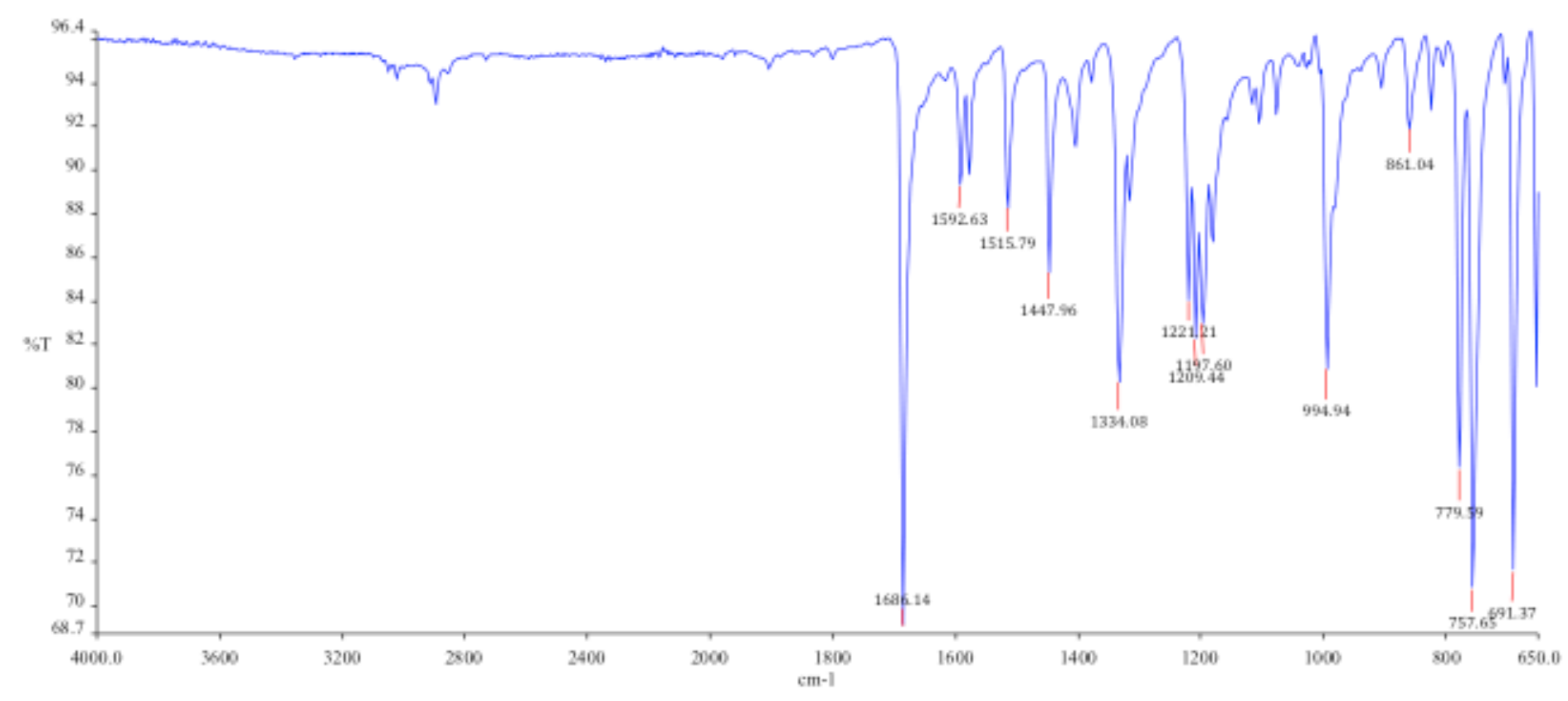

Figure S23: IR spectrum of 1c. 


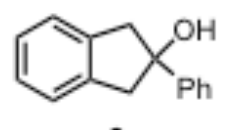

$2 a$

Date: Thursday, April 16, 2015

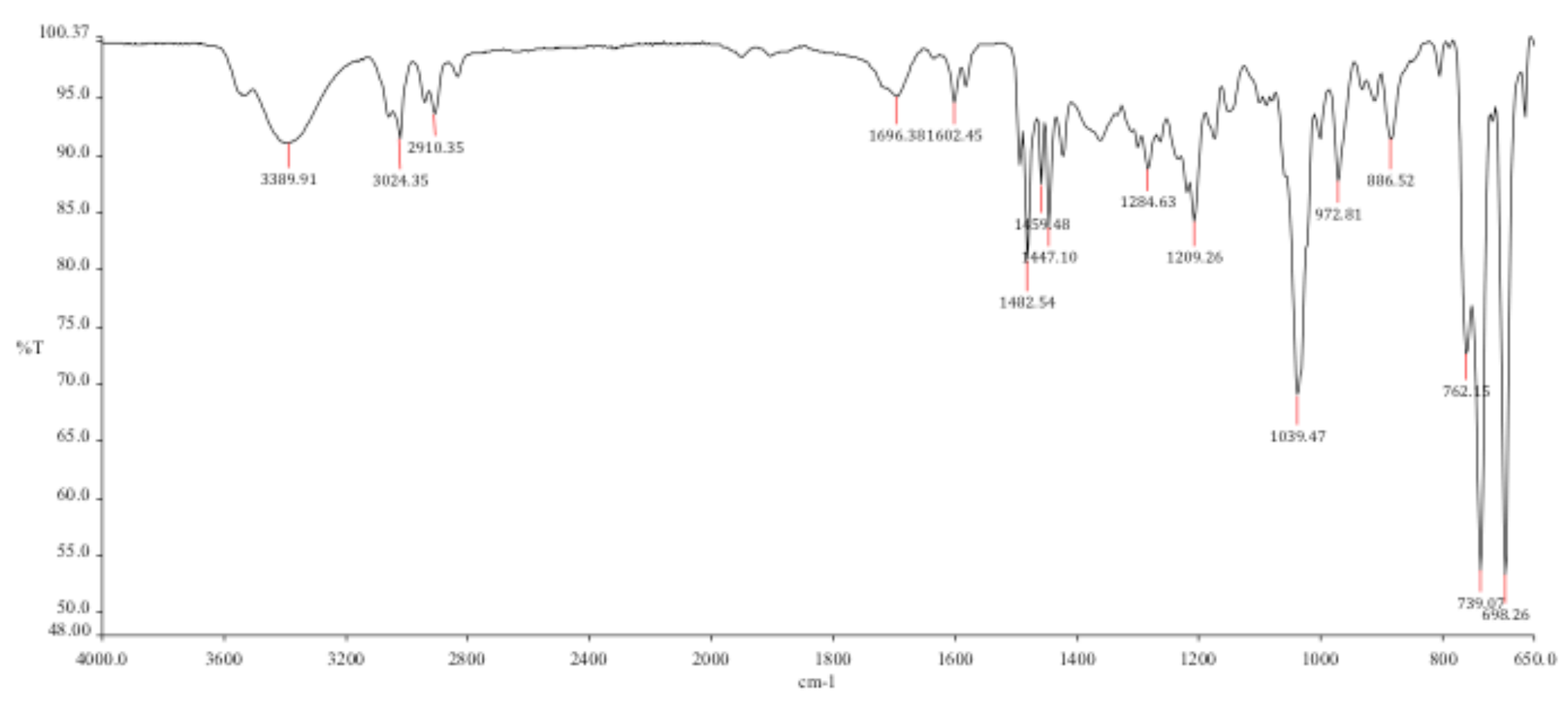

Figure S24: IR spectrum of $\mathbf{2 a}$. 


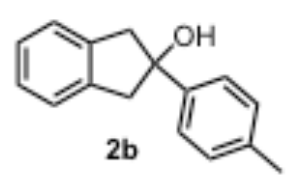

Date: Thursday, April 16, 2015

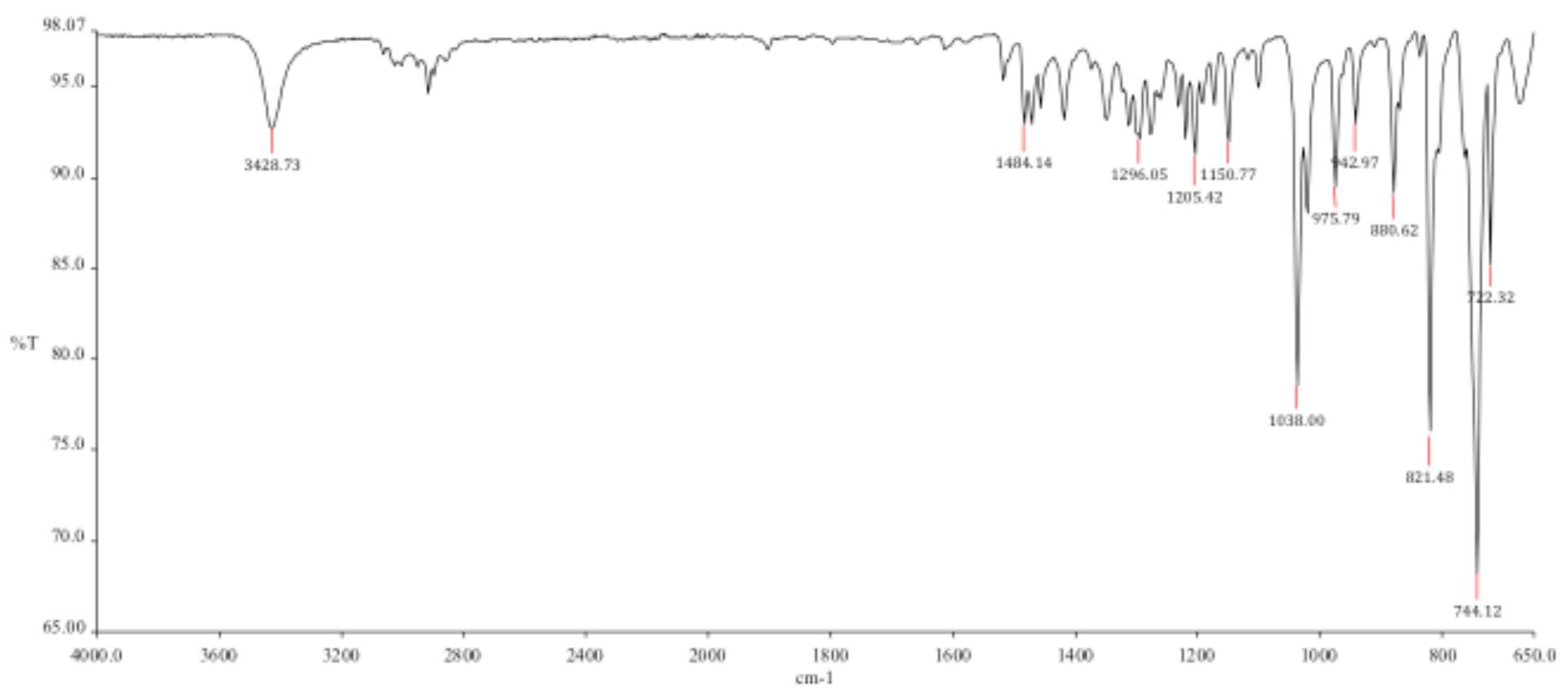

Figure S25: IR spectrum of $\mathbf{2 b}$ 


\section{REFERENCES}

(1) Grasa, G. A.; Colacot, T. J. Org. Process Res. Dev. 2008, 12, 522. 